\title{
When do response-related episodic retrieval effects co-occur with inhibition of return?
}

\author{
Matthew D. Hilchey ${ }^{1,2} \cdot$ Jason Rajsic ${ }^{3}$ Jay Pratt $^{1}$ \\ Published online: 27 April 2020 \\ (C) The Psychonomic Society, Inc. 2020
}

\begin{abstract}
At some point, spatial priming effects more faithfully reflect response selection processes than they do attentional orienting or sensory processes. Findings from the spatial cueing literature suggest that two factors may be critical: (1) the amount of identity processing that is required in order to respond correctly (feature-based response hypothesis), and (2) the amount of spatial processing that is required in order to respond correctly (space-based response hypothesis). To test the first hypothesis, we manipulated whether observers made single keypress detection or two-choice localization responses to serially presented stimuli in peripheral vision and whether stimulus identity information processing was necessary before responding. Responses were always slowest when the target location repeated, consistent with an attentional orienting bias independent of keypress responding (i.e., inhibition of return; IOR). The localization procedure revealed a subtle additional cost for changing the target location and repeating a response, consistent with a response-related episodic retrieval effect predicted by the Theory of Event Coding (TEC). Neither effect was modulated by the need to discriminate features. To test the second hypothesis, we made spatial processing indispensable to response selection by requiring a decision between a detection and localization response, depending on where the target appeared. IOR was eliminated for detection, but not localization, responses, consistent with the TEC. Collectively, the findings suggest that the amount of space-based, but not feature-based, processing that is required to determine a response is responsible for the response retrieval effects that can co-occur with IOR.
\end{abstract}

Keywords Attention $\cdot$ space-based $\cdot$ Inhibition of return $\cdot$ Priming

\section{Introduction}

How we perceive and act toward an item or event in our visual field has a considerable impact on how we will perceive and act on a subsequent item or event. Indeed, selection history (as it has come to be known) is one of the most significant drivers of human behavior, with research showing that it affects everything from low-level sensory processing, to perception, to attentional orienting, to making connections between stimuli and stimuli and responses, to decision-making and response

Matthew D. Hilchey

Matthew.hilchey@utoronto.ca

1 Department of Psychology, University of Toronto, 100 St. George St, Toronto, Ontario M5P 3G3, Canada

2 Rotman School of Management, University of Toronto, Behavioral Economics in Action at Rotman (BEAR), Toronto, Canada

3 Department of Psychology, Northumbria University, NE1 8ST, Northumberland Building, Newcastle upon Tyne, UK planning stages of processing (Allenmark, Müller, \& Shi, 2018; Burnham, 2018; Martín-Arévalo et al., 2019; Pascucci et al., 2019; Klein \& Redden, 2018; Koch, Frings, \& Schuch, 2018; Theeuwes, 2019; Valyear, Fitzpatrick, \& Dundon, 2019).

The present study is concerned with the selection history effects that occur in the cue-target sequences of the spatial cueing paradigm. This paradigm is marked by an elegant simplicity of experimental method but gives rise to seemingly inscrutable 40-year conundrums as to what the results from this method mean. In a typical paradigm, an initial attention-grabbing stimulus (the cue) appears abruptly somewhere in peripheral vision. This stimulus may or may not require a response. This stimulus is followed some time later by a second peripheral stimulus requiring a response (the target). The well-known selection history effects are the relative costs and benefits of randomly repeating versus switching the locations of the cues and targets These selection history effects are sometimes also called spatial priming effects, sequential dependencies or recency effects, and they are often interpreted broadly as reflecting biases of visual selective attention (Carrasco, 2011; Chica, Martín-Arévalo, Botta, \& Lupiánez, 2014; Klein, 2000, 2004, 2009; Maylor, 
1985; Maylor \& Hockey, 1985; Posner, 1980; Posner \& Cohen, 1984). Some variations of this procedure produce benefits when the second stimulus appears at the same location as the first (i.e., faster responses to the target), whereas others produce costs (i.e., slower responses to the target). These differences can occur even when the stimulus-onset asynchronies are held constant. What determines these polarity shifts and are these shifts necessarily emblematic of biases in visual selective attention?

Broadly speaking, chronometric studies show that the stimulus-response selection demands determine polarity shifts (i.e., whether benefits or costs are found; see, e.g., Adam \& Pratt, 2004; Christie \& Klein, 2001; Coward, Poliakoff, O'Boyle, \& Lowe, 2004; Dukewich, 2009; Eng et al., 2017; Guy, Buckolz, \& Pratt, 2004; Hilchey, Klein, \& Satel, 2014; Hommel, 1998, 2005, 2007; Ivanoff \& Klein, 2004; Kingstone \& Pratt, 1999; Klein, Wang, Dukewich, He, \& Hu, 2015; Lupiáñez \& Milliken, 1999; Lupianez, Ruz, Funes, \& Milliken, 2007; Lupiáñez, Martín-Arévalo, \& Chica, 2013; MacInnes, Krüger, \& Hunt, 2015; MartinArevalo, Chica, \& Lupianez, 2016; Pratt \& Abrams, 1999; Pratt, Adam, \& O'Donnell, 2005; Prime, Visser, \& Ward, 2006; Prime \& Jolicoeur, 2009a, 2009b; Taylor \& Donnelly, 2002; Taylor \& Klein (2000); Tanaka \& Shimojo, 1996, 2000; Terry, Valdes, \& Neill, 1994; Welsh \& Pratt, 2006; see Christie \& Klein, 2008; D'Angelo, Thomson, Tipper, \& Milliken, 2016; Frings, Schneider, \& Fox, 2015; Klein, 2004; Klein \& Redden, 2018; Lupianez, 2010, for reviews). For example, in some of our recent studies, we instructed people to make eye movements to sequentially presented stimuli before identifying their shapes with keypress responses (e.g., Hilchey, Antinucci, Lamy, \& Pratt, 2019; Hilchey, Rajsic, Huffman, Klein, \& Pratt, 2018). On the one hand, we found that eye movements were slower when the target location repeated relative to when it appeared elsewhere. This cost occurred regardless of whether the target's non-spatial features, like its color or shape, repeated. This observation is consistent with a phenomenon known as inhibition of return (IOR). Strictly speaking, IOR refers to a reluctance to orient attention to places that have been looked at or to which an eye movement had recently been primed and its presumed evolutionary function is to facilitate foraging (Hilchey et al., 2014; Posner, Rafal, Choate, \& Vaughan, 1985). On the other hand, the keypress responses showed both benefits and costs. When responses repeated, repeating the stimulus location led to benefits. When responses switched, repeating the stimulus location led to costs. These keypress findings are consistent with a framework called the Theory of Event Coding (TEC; Hommel, Müsseler, Aschersleben, \& Prinz, 2001).

The TEC accounts for the keypress effects by stipulating that the location of a stimulus and the response to it are linked together in implicit episodic memory to form a common, binary event representation (e.g., Hommel, 1998; 2005; Hommel, 2004, 2019; Memelink \& Hommel, 2013). If a stimulus location or response location later repeats, then the recently associated response location or stimulus location is retrieved, respectively. If the retrieved response or stimulus location is inconsistent with the required response or stimulus location, then some amount of interference occurs, which leads to relatively slow response times (RTs). If a stimulus location and response location both repeat or change, no inconsistent information is retrieved and thus no interference occurs, which leads to relatively fast RTs (Hommel, 1998, 2005, 2007). At the crux of the TEC is the notion that these relative RT differences can be accounted for strictly in terms of the partial overlap that exists between the current and retrieved event representation. In theory, all relative RT differences result from partial overlap. The idea is that the interference that is generated by partial overlap takes some time to resolve, thus leading to relatively slow responses. Reflecting this theory, the slower responses for repeating a target location but not a response, or vice versa, relative to repeating or switching both is referred to as a partial repetition cost (PRC). Effects of partially repeating a recent stimulus location and response - or PRCs - may be referred to more broadly and neutrally as visuomotor repetition effects (e.g., Hommel \& Colzato, 2009).

The TEC cannot account for IOR (i.e., any RT difference that does not result from partial stimulus location-response overlap) and visuospatial attention theories cannot account for PRCs (i.e., any RT difference that does result from partial stimulus location-response overlap). Regardless, there is no real theoretical conflict here. Our studies suggest that both classes of theory are viable. PRCs and IOR are not mutually exclusive; they occur at demonstrably different stages of processing. Specifically, there are intrinsic orienting biases against previously oriented to locations, consistent with seminal research on IOR. Visuomotor repetition effects, or PRCs, kick in after these initial shifts of attention. The PRCs are intimately related to relatively late response-selection processes (Hilchey, Pratt, \& Lamy, 2019; see e.g., Christie \& Klein, 2001; Klein, 2004, for other multi-stage theories; see also Sanders, 1998, for a review of many other relevant multistage theories dating back to the 1960s). Despite this, we are still faced with problems: we do not know exactly when to expect visuomotor repetition effects generally, or the PRCs predicted by the TEC more specifically. While there is no doubt that the TEC can account for a wide range of visuomotor repetition effects related to stimulus location and response repetition with its partial mismatching principles (Hommel, 2019; Hommel, Proctor, \& Vu, 2004; Hommel \& Wiers, 2017), the necessary and sufficient conditions for revealing PRCs remain poorly specified.

What we do have from the literature is evidence that PRCs are not obligatory (e.g., Hommel, 1998, 2007; Huffman, Hilchey, Weidler, Mills, \& Pratt, in press; Memelink \& Hommel, 2013; Tanaka \& Shimojo, 1996; Welsh \& Pratt, 2006). There is no mandatory coupling between response 
repetition and stimulus location retrieval or between stimulus location repetition and response retrieval. Some of the most basic research on visual orienting in the spatial cueing paradigm is sufficient for showing this. Consider very basic spatial cueing paradigms in which a single button must be pressed to serially presented stimuli (so-called detection tasks; e.g., Schopper, Hilchey, Lappe, \& Frings, 2019; Tanaka \& Shimojo, 1996). Here, stimulus locations randomly repeat or switch and the response stimulus intervals are somewhere between a few hundred milliseconds and a few seconds. Left unqualified, the TEC predicts that responses should be faster when the target location repeats instead of switches. This is because a PRC should theoretically occur when the target location changes (and response repeats) whereas it cannot occur when the target location repeats (and response repeats). Instead, much of the literature shows the opposite result (IOR; e.g., Maylor \& Hockey, 1985; Huffman, Hilchey, \& Pratt, 2018, for review) and yet, not all studies converge. Some closely related studies show no evidence of IOR at all (e.g., Wilson, Castel, \& Pratt, 2006).

The basic goal for this study is to sort out when visuomotor repetition effects, at the level of stimulus location and response repetition, are likely to occur and thus overshadow IOR. The first experiment tests what we call the featurebased response hypothesis (see also Taylor \& Ivanoff, 2005, for similar ideas). Across a number of investigations, we have found that tasks involving responding based on the identity of a stimulus are effective at eliciting visuomotor repetition effects (e.g., Hilchey, Rajsic, Huffman, \& Pratt, 2017a; Hilchey, Rajsic, Huffman, \& Pratt, 2017b; Hilchey, Rajsic, et al., 2018; see also Rajsic, Bi, \& Wilson, 2014; Wilson, Castel, \& Pratt, 2006). This hypothesis is informed as well by a range of findings from spatial cueing, spatial negative priming, and visual search procedures (see also: Hilchey, Pratt, \& Lamy, 2019; Huffman et al., 2018; Hilchey, Leber, \& Pratt, 2018; Huffman et al., in press, for further discussions). In such cases, repeating the target location results in a benefit when the response repeats and a cost when the response changes. Unlike with the very basic spatial cueing paradigms used to study IOR (e.g., Maylor \& Hockey, 1985; Kwak \& Egeth, 1992), with these procedures there are substantial non-spatialprocessing demands, and in both cases response repetition leads to just the sort of results predicted by the TEC.

The feature-based response hypothesis is mainly a datadriven hypothesis, but it may also be helpful to consider briefly how this hypothesis could be viable theoretically. One possibility is that feature-based stimulus-processing modes lead to object permanence assumptions that are critical for the retrieval of event representations (Hilchey, Leber, \& Pratt, 2018). The basic line of reasoning is that, outside of the laboratory, visuomotor systems learn that objects do not usually spontaneously disappear and reappear with different identities. It may be the case that an object permanence assumption is only in effect when object processing (i.e., non-spatial processing) becomes necessary or useful for deciding upon a response. If the assumption is being made, then the visuomotor system may infer that stimulus location repetitions usually signal that the recently associated object is present (and that the recent response remains appropriate) and that response repetition usually signals the recently associated object (and the location at which it recently occurred). A prediction error essentially occurs when there is a mismatch, leading to interference and a cognitively demanding resolution process. If there is no object-processing requirement (i.e., color, shape, category processing, and so on), then this assumption is simply not made and, accordingly, the visuomotor repetition effects do not occur. When the assumption is being made, note that all visuomotor repetition effects follow logically from the partial mismatching principles of TEC. If a stimulus location retrieves the object contingency, then the system implicitly predicts that the recently associated response remains appropriate. If a response retrieves the object contingency, then the system implicitly predicts that the recently associated stimulus location remains appropriate. Interference occurs when there is a partial mismatch. In fact, this is just generally how the TEC attempts to explain so-called combined or subjective expectancies (e.g., Hommel et al., 2004; Kingstone, 1992; Müller \& Krummenacher, 2006; Notebaert \& Soetens, 2003; Soetens, Boer \& Hueting (1985); Soetens, 1998; Töllner, Gramann, Müller, Kiss, \& Eimer, 2008).

The key problem that this first experiment aims to solve is whether the feature-based processing associated with stimulus identification is in fact a necessary precondition for observing visuomotor repetition effects. The TEC more or less consistently argues that a proviso along this line is needed to predict visuomotor repetition effects involving identity repetition (Hommel, 1998; Hommel, Memelink, Zmigrod \& Colazto (2014); Memelink \& Hommel, 2013). Be that as it may, it is worth noting that the TEC rarely, if ever, invokes featurebased processing modes as the sine qua non for visuomotor repetition effects involving stimulus location and response repetition (Hommel, 2005, 2007).

The second experiment tests what we call the space-based response hypothesis. This hypothesis was motivated by a few observations and some theory. First, foreshadowing the results of our first experiment, there was no empirical support for the feature-based response hypothesis. Thus, some other factor must necessarily be responsible for generating visuomotor repetition effects that can overshadow IOR. Second, we took a closer look at the findings of Wilson, Castel, \& Pratt, (2006). Wilson et al. (2006) found no evidence of IOR when detection responses were required to sequentially presented targets, which is an unusual result for keypress detection procedures in the visual modality (Huffman et al., 2018). While we initially suspected that IOR was abolished because their procedure, unlike most others, involved a go/no-go component (the 
detection response was contingent on the color of the stimulus, i.e., the feature-based response hypothesis), we later realized that their procedure also allowed for more elaborate space-based processing than is typical. This is because stimuli falling on the horizontal midline alternated predictably between go and no-go, which means that increased spatial processing was especially useful for choosing whether and when to respond. Third, and more theoretically, Hommel (2007) has proposed specifically that links between responses and stimulus locations - or event files including response and stimulus locations - are most likely to be retrieved when deemed relevant for choosing between spatially-defined response choices. So long as no response in Wilson et al. (2006) is considered to be part of the response choice set, their findings are broadly consistent with this proposal.

\section{Experiment 1}

We started off by testing the feature-based response hypothesis. If the feature-based response hypothesis is correct, then it should be possible to generate visuomotor repetition effects involving stimulus location and response repetition by simply adding non-spatial processing requirements to standard keypress detection procedures. More specifically, if featurebased processing is a necessary and sufficient qualifier for all PRCs predicted by the TEC, then it should be possible to increase or generate PRCs by adding a non-spatial processing requirement. To accomplish this, the feature-based response hypothesis was tested in procedures with two different response execution requirements. One involved making single keypress detection responses to sequentially presented targets, as in Wilson et al. (2006). The other involved deciding between a left or right response depending on whether a target appeared on the left or right side of a central fixation stimulus, as in Taylor and Donnelly (2002). While a localization procedure is not strictly necessary for testing the hypothesis, it provides an additional baseline to test for PRCs in the detection procedure. Our localization procedure is also able to provide evidence for PRCs in and of itself - more on this below.

In both procedures, targets appeared randomly on the vertices of an imaginary square centered on fixation. Target colors could randomly repeat or change. The key manipulation within each of these procedures was whether the color of these targets had to be processed before the appropriate response could be made to it (i.e., feature-based processing). In one version, which we call "standard," responses were to be made to every stimulus, regardless of color. The processing requirements of this procedure thus match the conditions under which IOR is observed and PRCs are not. In the other version, it was necessary to process color information before making a response because responses were to be withheld to one color but not to others (i.e., a go/no-go task, as in Wilson et al., 2006). In all cases, between these peripheral targets, a small white circle appeared at fixation. This is sometimes called a cue-back because it theoretically helps to ensure that attention is re-oriented back to center, a neutral location, in between peripheral targets (MacPherson, Klein, \& Moore, 2003; Prime et al., 2006; Pratt \& Fischer, 2002). We go beyond this here by requiring that the participants acknowledge this cue-back by responding to it with all keys in the procedure. ${ }^{1}$

The standard target-target detection procedure, which does not require responses to be formed on the basis of stimulus identity, will reveal some form of IOR, consistent with many other studies. Put simply, responses will be slower when the target location repeats instead of switches. These RT patterns should change in the go/no-go version. If adding a featureprocessing demand generates visuomotor effects, or PRCs specifically, then the cost of repeating versus switching the target location should become smaller when it becomes necessary to process stimulus identity information. This is specifically because any cost for repeating the target location (i.e., IOR) should be counteracted by a PRC when the target location changes (and response repeats) if the PRC depends on feature processing.

The standard localization procedure will also show some form of IOR, consistent with many other studies. More important, with this procedure, we can also test directly for PRCs with and without the feature-based processing demands. This is because we used four locations paired with two responses. With these 2:1 stimulus location-response mappings, a PRC can theoretically arise when the target location switches but the response repeats. PRCs are tested for by comparing performance on the location switch-response repeat transition to performance on the location switch-response switch transition that is equidistant from the previous target location. This helps ensure that any distance-dependent visuospatial effect against the previous target location is equal between the two transition types (e.g., Hilchey, Pratt, \& Lamy, 2019). If feature-based processing generates PRCs, then a PRC would be expected when colors must be processed to correctly respond (i.e., the go/no-go version), but not when color processing is irrelevant to responding. If feature-based processing mediates PRCs

\footnotetext{
${ }^{1}$ Readers may wonder whether the magnitude of the PRCs related to location and response repetition are affected by these intervening response events; they are not (Hilchey et al., 2017a, b). According to the TEC, this may not be surprising. Think of it this way: all responses involved in the task must be made to these intervening events. Thus, the response required to an ensuing peripheral target necessarily retrieves the recently associated intervening event location (the central location in this case). This means that retrieval should occur regardless of whether a peripheral target location repeats or switches. Assuming, as the TEC does, that event file representations are binary and independent, this retrieval should affect peripheral target location repeat and switch transitions equally by slowing down overall response time (i.e., there should be a retrieval effect that leads to interference, but it should apply equally to peripheral target location repeat and switch transitions, as in Hilchey et al. (2017b)).
} 
without being fundamentally responsible for them, then a PRC would be expected in both versions, with its magnitude being larger in the version with the added feature-processing demand.

Another test of a PRC is made possible by evaluating whether the cost of repeating the target location relative to switching it to the maximally distant location is smaller in the detection than the localization procedure. There is theoretically no potential for a PRC at either of these two levels of target location transition in the localization procedure. This is because the target location and response either repeat together or switch together. There is, however, a potential for a PRC in the detection procedure. This PRC should occur specifically when the target location changes because here the response must also repeat (i.e., a partial repetition). Considered together, any cost for repeating the target location should be smaller in the detection than the localization procedure, since the PRC unique to the detection procedure would help to offset IOR.

Finally, while secondary to our main objectives, our methods also afford us an opportunity to show that repeating versus switching target colors has negligible impacts on target location transition effects in the standard procedures (Huffman et al., 2018, for review). We will also show little to no role for color repetition in modifying these effects even when it is necessary to process target color, which has also been shown before (e.g., Hilchey et al., 2017a; Hommel, 1998; Wilson et al., 2006).

\section{Method}

Participants Forty-eight undergraduates from the University of Toronto consented to participate for course credit (M age $=19.5$ years; four left-handed; 13 males). These sample sizes should be more than adequate for consistently $(>80 \%$ of the time) revealing IOR and PRCs in standard procedures at conventional alpha levels for statistical significance (0.05).

Our logic is as follows: We estimate that the magnitude of IOR is roughly $20 \mathrm{~ms}$ in keypress detection and localization procedures (see Huffman et al., 2018; specifically, Fig. 1 and Appendix). We estimate from our earlier experiments involving localization responses (Hilchey, Pratt, \& Lamy, 2019) and discrimination responses (Hilchey et al., 2017a, 2017b) that the magnitude of the PRC is roughly $20 \mathrm{~ms}$. We know from our earlier work with keypress detection and localization procedures (e.g., Hilchey et al., 2019; Huffman, Hilchey, \& Pratt, 2018; Huffman, Hilchey, Weidler, Mills, \& Pratt, 2020 ) that the correlation between the repeated measures of target location transition is strong ( $>.9$; see also Hilchey et al., 2019), but we assume .9. We expect that the correlation between the repeated measures of each task (e.g., go/no-go vs. standard) will be much greater than the known correlation between two-choice localization procedures and two-choice feature discrimination procedures (0.6; as reported by Hilchey, Pratt, \& Lamy, 2019, and as estimated from the data in Huffman et al., 2018). We expect this because the stimulusresponse translation rules are held constant between task levels (i.e., go/no-go vs. standard) of the current procedures whereas these rules were different for the keypress localization and discrimination procedures from which we originally estimated this correlation. We expect the correlation to be at least 0.7 , but will assume 0.7 . The standard deviation on the RTs at any given level in these designs is likely to be around $50 \mathrm{~ms}$ (see Huffman et al., 2018, Appendix) but it may approach $65 \mathrm{~ms}$ in some cases (as reported by Hilchey, Pratt, \& Lamy, 2019). We will estimate the sufficient sample size assuming 50 $\mathrm{ms}, 55 \mathrm{~ms}, 60 \mathrm{~ms}$, and $65 \mathrm{~ms}$ standard deviations.

We determined the sample sizes that would be required to achieve $80 \%$ power at an alpha level of 0.05 for a main effect of location transition and an interaction between location transition and task using GLIMMPSE's open source software (Kreidler et al., 2013). According to our estimates, somewhere between seven and 16 participants should be sufficient.

Stimuli and apparatus The experiment took place in a dimly lit room. Stimuli were displayed on 24-in. LED monitors, using 2,560 $\times 1,440$ resolution graphics mode and a $120-\mathrm{Hz}$ refresh rate, at a constant viewing distance of $57 \mathrm{~cm}$, which was ensured by a chinrest. The monitors were connected to Dell computers, with the presentation of all stimuli and the collection of responses being controlled by a custom Python program. Responses were made on a computer keyboard.

The monitor's background was black (luminance $=$ $0.28 \mathrm{~cd} / \mathrm{m}^{2}$ ). The fixation stimulus was a white (luminance $\left.=194.83 \mathrm{~cd} / \mathrm{m}^{2}\right)$ cross $\left(0.30^{\circ} \times 0.30^{\circ}\right.$ of visual angle) at screen center. Five unfilled gray (luminance $=$ $\left.31.67 \mathrm{~cd} / \mathrm{m}^{2}, \mathrm{x}=0.31, \mathrm{y}=0.34\right)$ placeholder squares $\left(2.00^{\circ} \times 2.00^{\circ}\right.$; linewidth $=1$ pixel $)$ marked the locations at which response stimuli ("targets") could occur. One such square was at screen center; the remaining four were positioned on the vertices of an imaginary square $\left(5.30^{\circ} \times\right.$ $5.30^{\circ}$ ), which was centered on fixation. The distance between the fixation cross and the center of each peripherally placed placeholder square was $7.50^{\circ}$.

In the visual periphery, one placeholder square at a time could be filled in with red (luminance $=9.62 \mathrm{~cd} /$ $\mathrm{m}^{2}, \mathrm{x}=0.63, \mathrm{y}=0.34$ ), green (luminance $=33.66 \mathrm{~cd} /$ $\mathrm{m}^{2}, \mathrm{x}=0.31, \mathrm{y}=0.63$ ), or blue (luminance $=2.86 \mathrm{~cd} / \mathrm{m}^{2}$, $\mathrm{x}=0.16, \mathrm{y}=0.05)$, and, depending on the conditions ( $\mathrm{go} /$ no-go or standard, below), responses were made to these. On each trial, the fixation cross transformed into a white circle $\left(\right.$ radius $=0.25^{\circ}$ ) in between the appearance of each color in the visual periphery and had to be responded to by pressing all keys required by the task simultaneously. 
Go/No-Go Localization Procedure (attention to color required)

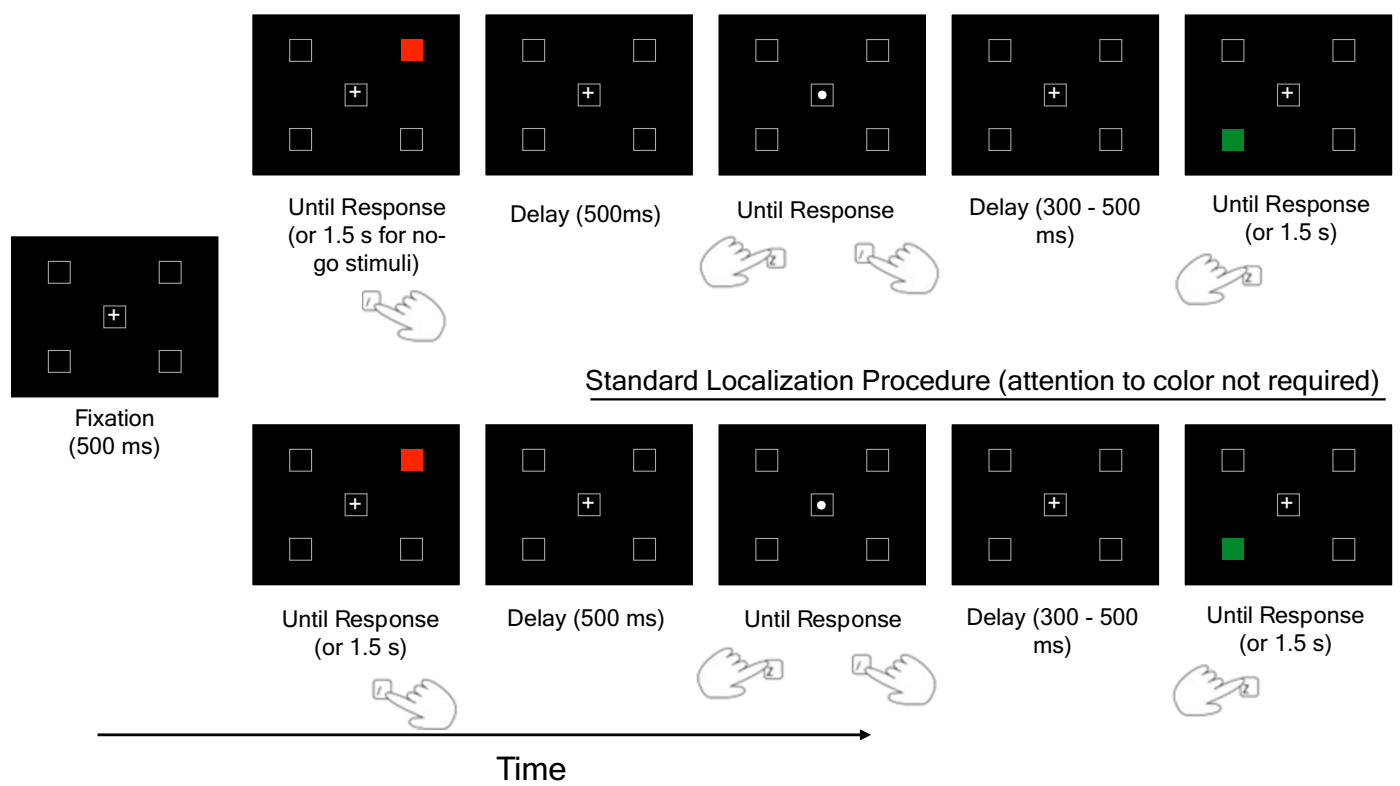

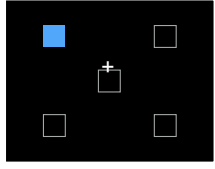

Rare no-go color
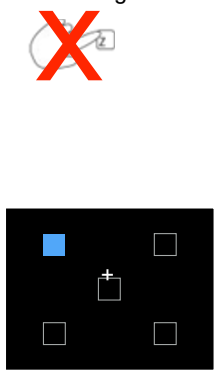

Rare go color

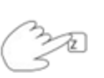

Fig. 1 A possible trial sequence from the localization procedures (go/nogo top panel, standard bottom panel). Colored squares that appear randomly in the left and right visual hemifield are localized with left and right keypress responses, respectively. The colored squares are separated by a bimanual response event at fixation. The Go/No-Go and Standard procedures are identical, except responses are not made to the

Procedure and design Each trial began with the presentation of the placeholder squares, followed $500 \mathrm{~ms}$ later by the onset of the fixation cross. Participants were instructed to remain fixated on the cross. Five hundred ms after the onset of the cross, a random peripheral placeholder turned red, green, or blue. Participants were instructed to use their peripheral vision to process this stimulus, which remained onscreen until response (or $1.5 \mathrm{~s}$ had elapsed, see below). Five hundred $\mathrm{ms}$ after the offset of the colored square, a white circle replaced the fixation cross, which remained onscreen until response, after which it transformed back into a cross. Randomly either 300 or $500 \mathrm{~ms}$ after the reappearance of the fixation cross, a random peripheral placeholder turned red, green, or blue, which remained onscreen until response (or $1.5 \mathrm{~s}$ had elapsed). If an error was made during the trial, the offset of this stimulus was followed by an instruction screen that reminded participants of the response requirements; this instruction screen remained until acknowledged by a keypress. Regardless of error, there was a 1-s blank interval between all trials, during which no stimuli were onscreen.

Half of the participants were assigned to the detection response procedures whereas the other half were assigned to the localization response procedures. Within each procedure, each participant performed one version of it in which it was necessary to attend to color information in the visual periphery (go/ no-go) and another in which it was not (i.e., the standard detection or localization procedure), the order of which was counterbalanced across participants. rarely occurring color in the Go/No-Go procedure (which just happens to be blue in this example and is removed from the trial sequence). The rare color may randomly appear as either the first, second, both, or neither (illustrated) colored square in the trial. In these examples, the target color, response, and location (change diagonal transition) have switched

In the localization procedures, one of the three possible colored squares in the visual periphery was selected at random to appear less often than the other two, which occurred equally often. The rare color appeared on only $17.24 \%$ of trials as the first colored square (40\%), second colored square (40\%), or both (20\%). In the go/no-go version, this rare stimulus served as the no-go stimulus (i.e., do not respond to it), whereas in the standard localization procedures, responses were made to it (i.e., responding was not conditional on color processing). No-go stimuli remained onscreen until $1.5 \mathrm{~s}$ elapsed or a response was made, whereas go stimuli remained onscreen until a response was made. For each participant, the same rare color that was used for the go/no-go version was also used as the rare color for the standard localization procedure. Localization procedures are illustrated in Fig. 1.

In the go/no-go version of the standard detection procedure, one of the three possible colored squares in the visual periphery was again selected at random to appear less often than the other two, which occurred equally often. As in the localization procedures, the rare color appeared on only $17.24 \%$ of trials as the first colored square $(40 \%)$, second colored square $(40 \%)$, or both (20\%). In the standard detection procedure, instead of rare colors, on $17.24 \%$ of trials the first color square $(40 \%)$, second color square $(40 \%)$, or both $(20 \%)$ were omitted. These trials in which a colored squared did not appear are known as "catch trials," and are intended to guard against anticipatory (i.e., non-stimulus driven) responses to colored targets. 
In the standard detection procedure, the spacebar (right index finger) was pressed to each colored square and white circle, whereas in the go/no-go version of this the spacebar response was withheld to the rare color. In the localization procedure, colored squares that appeared to the left and right of fixation were localized with ' $z$ ' (left index finger) and '/' (right index finger) keypress responses, respectively, whereas in the go/no-go version of this these responses were withheld to the rare color. In the localization procedures, both response keys were pressed simultaneously to the white circle at fixation, whereas in the detection procedures only the spacebar response was required to the white circle at fixation.

In all cases, across all versions, participants were explicitly instructed that there was no relationship between the location of the first and second colored square in a trial. They were further instructed that these were simple RT tests and that they should do their best to react as quickly and as appropriately as possible to each stimulus. In the standard detection and localization procedures, it was stressed that color processing was unnecessary for successfully completing the task (i.e., the mere detection of an abrupt onset in the visual periphery would be sufficient). In the go/no-go versions, it was stressed the color processing was imperative for successfully completing the task (the participant was told not to respond to a particular color (i.e., the rare color)).

Each version of each procedure consisted of 20 practice trials followed by 464 experimental trials, within which the location and color of the peripheral targets randomly repeated. As noted, each participant completed both versions of either the detection or localization procedures.

\section{Results}

Localization procedures In the go/no-go version, the mean false-alarm rates to the first and second peripheral targets in the trial were $19.5 \%$ (range $=0.0-50.0 \% ; \mathrm{SD}=16.1$ ) and $17.9 \%$ (range $=0.0-52.1 \% ; \mathrm{SD}=13.8$ ), respectively. For the subsequent RT analyses, regardless of procedure, all trials involving a rare color were excluded. This means that all trials involving a no-go stimulus were excluded in the go/no-go version and that trials involving the rare color were excluded in the standard procedure. This exclusion was necessary to make the color transitions between the go/no-go and standard version identical. An additional $0.6 \%$ of trials from the remaining data were excluded for exceptionally slow responses $(>3 \mathrm{~s}$ ) to either the first peripheral target, the center circle, or the second peripheral target. We assumed in these cases that the participant disengaged from the task.

For the remaining data, the mean error rates (\%) to the first and second peripheral target were near ceiling in both tasks. For the go/no-go version, the mean error rates to the first and second peripheral targets were $0.7 \%$ and $0.5 \%$, respectively. For the standard version, the mean error rates to the first and second peripheral targets were $1.0 \%$ and $2.0 \%$, respectively. These low rates precluded a meaningful statistical analysis of errors, as many participants did not make mistakes in various cells of the design. All trials containing errors were removed from the subsequent RT analyses. Finally, we excluded as outliers all trials with RTs to the second peripheral target less or greater than 3 standard deviations from the participant's mean in both tasks, which resulted in the loss of $1.7 \%$ of the remaining data.

Effects of repeating/changing the locations and colors of peripheral targets on RTs were analyzed with a 4 (location transition: repeat, change vertical, change horizontal, change diagonal) $\times 2$ (color transition: repeat or change) $\times 2$ (task: go/no-go or standard) repeated-measures analysis of variance (ANOVA). ${ }^{2}$ Figure 2 illustrates the mean RTs across all levels of the experiment.

The main effect of location transition was significant, $\mathrm{F}(3$, 69) $=15.06, p<0.01, \eta_{p}^{2}=0.40$. A planned comparison revealed that responses were slower when target location repeated $(355 \mathrm{~ms})$ instead of changed to the diagonal location $(338 \mathrm{~ms} ; \mathrm{t}(23)=5.64, \mathrm{p}<0.01, \mathrm{M}$ diff $=17 \mathrm{~ms} ; 95 \%$ confidence interval $(\mathrm{CI})=11-24 \mathrm{~ms}$ ), consistent with IOR. A second planned comparison revealed that responses were slower for the change vertical $(349 \mathrm{~ms})$ than change horizontal transition $(341 \mathrm{~ms} ; \mathrm{t}(23)=2.30, \mathrm{p}=0.03, \mathrm{M}$ diff $=8 \mathrm{~ms} ; 95 \% \mathrm{CI}=$ 1-15 ms), consistent with a PRC.

The main effect of color transition was significant, $F(1,23)$ $=5.356, p=0.03, \eta_{p}^{2}=0.19$, with slightly faster responses for color repeats $(344 \mathrm{~ms})$ than switches $(347 \mathrm{~ms})$. The main effect of task was also predictably significant, $\mathrm{F}(1,23)=$ 44.62, $\mathrm{p}<0.01, \eta_{\mathrm{p}}^{2}=0.66$, with faster responses in the standard localization procedure $(322 \mathrm{~ms})$ than in the go no-go procedure (369 ms).

There was a significant interaction between task and color transition, $F(1,23)=7.07, p=0.01, \eta_{p}^{2}=0.23$. In the go nogo version, responses were faster when the target color repeated $(367 \mathrm{~ms})$ instead of switched $(371 \mathrm{~ms} ; \mathrm{t}(23)=-2.78, \mathrm{p}=$ $0.01, \mathrm{M}$ diff $=-5 \mathrm{~ms} ; 95 \% \mathrm{CI}=-8-(-1) \mathrm{ms})$. In the standard localization version, there was no statistical difference between target color repeats $(322 \mathrm{~ms})$ and switches $(323 \mathrm{~ms}$; $\mathrm{t}(23)=0.59, \mathrm{p}=0.55, \mathrm{M}$ diff $=-1 \mathrm{~ms} ; 95 \% \mathrm{CI}=-3-(-1)$ $\mathrm{ms})$. None of the remaining interactions approached statistical

\footnotetext{
${ }^{2}$ Some participants in the go/no-go version made false alarms on many no-go trials (e.g., $51 \%, 43 \%, 36 \%$ ). This raises the possibility that some participants may not have consistently processed target color in this condition, which could explain why the interaction between location transition and task was not significant. To provide more conservative tests, we ran this ANOVA with four different participant exclusion criteria for the false-alarm rates in the go/no-go version. Any participant who made false alarms on over $50 \%, 40 \%, 30 \%$, and then $25 \%$ of the trials was excluded from the respective ANOVA. These resulted in the exclusion of one, two, five, and nine participants, respectively. There were significant main effects of location transition and task across all analyses (all ps $<0.01$; all in the expected directions). The main effect of color transition was always significant $(\mathrm{p}<0.05)$ or marginally significant $(\mathrm{p}<$ 0.10 ). The interaction between task and color transition was always significant (ps < 0.05). None of the analyses provided any support for the remaining interactions (all ps >.1).
} 


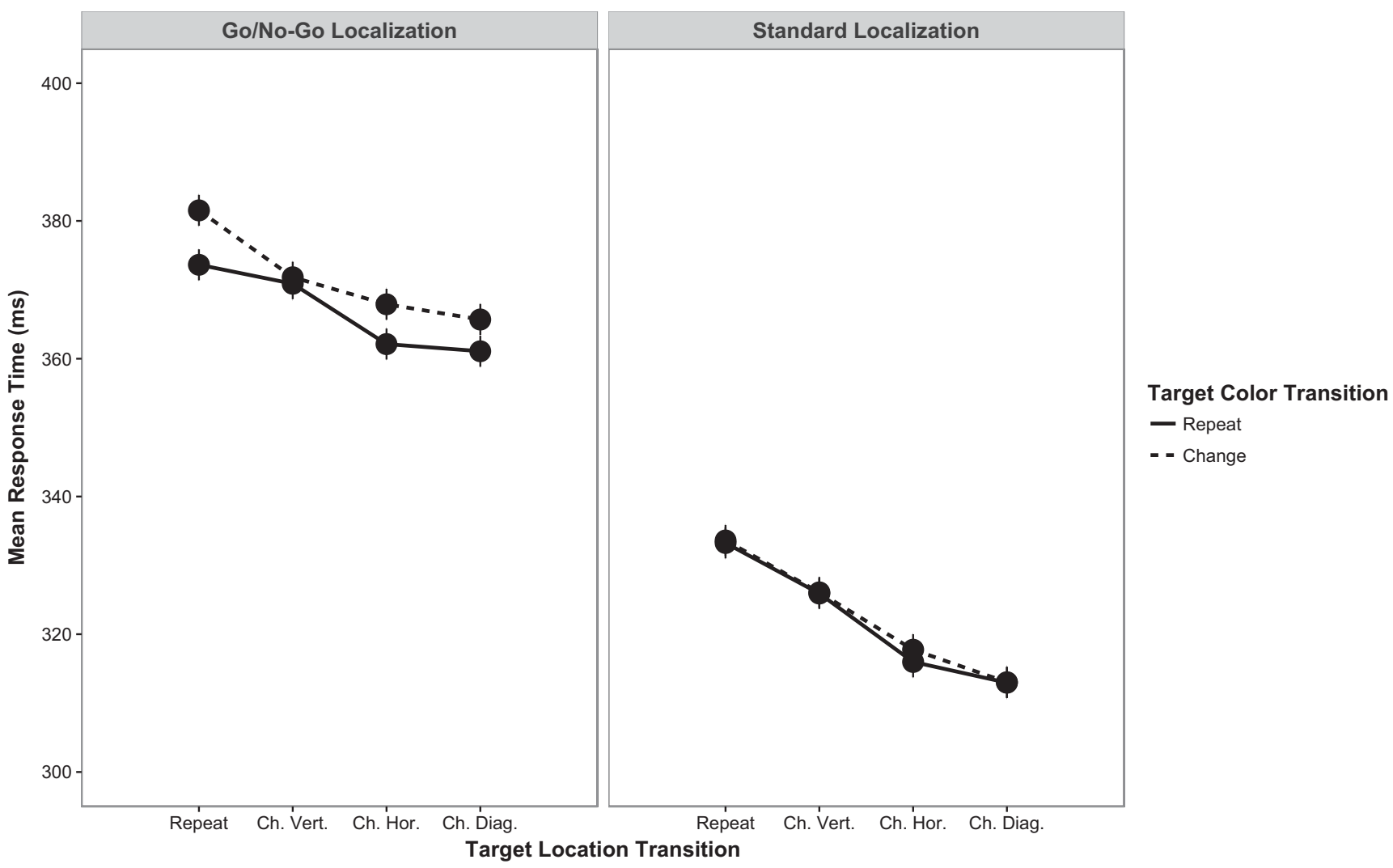

Fig. 2 Mean response times for all combinations of target location transition, target color transition, and task (columns) of the localization procedure in Experiment 1. Error bars are half Fisher's Least Significant Difference (FLSDs) computed from the mean squared error term of the interaction

significance (location transition $\times$ task, $\mathrm{F}(3,69)=1.751, \mathrm{p}=$ $0.17, \eta_{\mathrm{p}}^{2}=0.07$; all other Fs $\left.<1\right)$.

Detection procedures In the go/no-go version, the mean false-alarm rates to the first and second peripheral target were $23.4 \%$ (range $=2.0-77.0 \% ; \mathrm{SD}=19.0)$ and $26.1 \%$ (range $=6.3-70.1 \% ; \mathrm{SD}=16.9$ ), respectively. In the standard version, the mean false-alarm rates to the absence of the first and second peripheral target were $10.7 \%$ (range $=0.0-66.7 \% ; \mathrm{SD}=15.7$ ) and $18.4 \%$ $($ range $=0.0-87.5 \% ; \mathrm{SD}=20.9) .{ }^{3}$ In the go/no-go version, all trials involving a no-go stimulus were excluded. In the standard version, all trials without a first or second target were excluded. An additional $0.2 \%$ of trials from the remaining data were excluded for

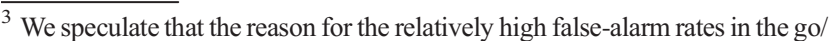
no-go version is because the appearance of the stimulus primed the most frequent response that was made to it whereas the absence of the stimulus (in the standard version) was not associated with a response and thus could not prime. In both versions, the interval between each stimulus offset and stimulus onset was roughly predictable, which likely increased the probability of anticipatory responses in both versions. Thus, overall, the false-alarm rate should be higher in the go/no-go version (anticipatory response tendency + response priming) than in the standard version (anticipatory response tendency alone).
}

exceptionally slow responses ( $>3$ s) to at least one of the three response stimuli within the trial. For the subsequent RT analysis, we excluded as outliers all trials with RTs to the second peripheral target less or greater than 3 standard deviations from the participant's mean in both tasks, which resulted in the loss of $1.5 \%$ of the remaining data.

Again, effects of repeating/changing the locations and colors of peripheral targets on RTs were analyzed with a 4 (location transition: repeat, change vertical, change horizontal, change diagonal) $\times 2$ (color transition: repeat or switch) $\times 2$ (task: go/no-go or standard) repeatedmeasures ANOVA. ${ }^{4}$ Figure 3 illustrates the mean RTs across all levels of the experiment.

The main effect of location transition was significant, $\mathrm{F}(3,69)=28.71, \mathrm{p}<0.01, \eta_{\mathrm{p}}^{2}=0.55$. RTs decreased with greater distance between the first and second colored target (same location $=352 \mathrm{~ms}$; change vertical $=$ $339 \mathrm{~ms}$; change horizontal $=338 \mathrm{~ms}$; change diagonal $=$ $333 \mathrm{~ms}$ ), consistent with IOR.

The main effect of color transition was significant, $F(1,23)$ $=6.351, p=0.02, \eta_{p}^{2}=0.22$, with slightly faster responses for color repeats $(339 \mathrm{~ms})$ than switches $(342 \mathrm{~ms})$. The main effect of task was also significant, $\mathrm{F}(1,23)=28.12$, $\mathrm{p}<$ 


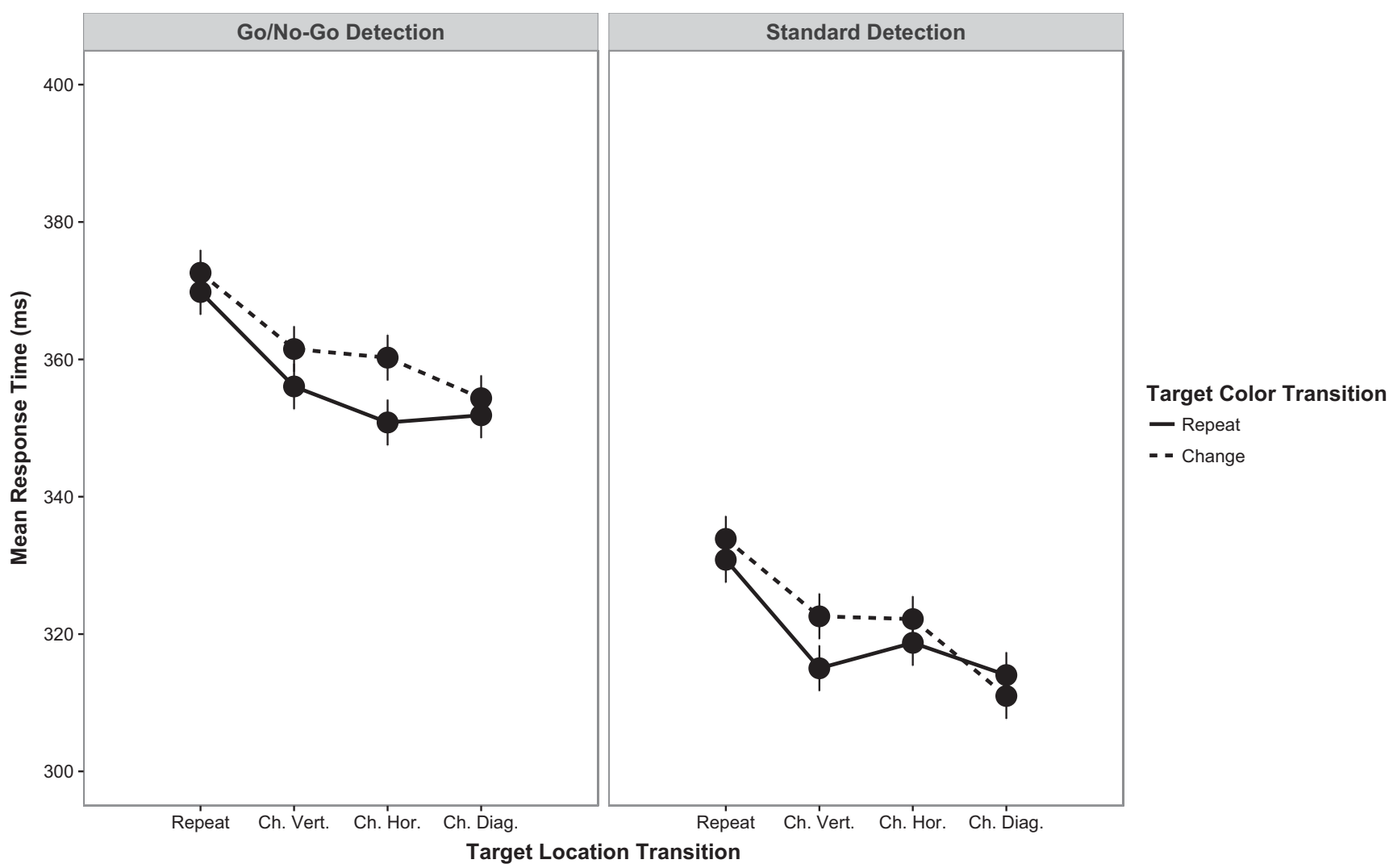

Fig. 3 Mean response times for all combinations of target location transition, target color transition and task (columns) of the detection procedure in Experiment 1. Error bars are half Fisher's Least Significant Difference (FLSDs) computed from the mean squared error term of the interaction

$0.01, \eta_{\mathrm{p}}^{2}=0.55$, with faster responses in the standard version $(321 \mathrm{~ms})$ than in the go/no-go version $(360 \mathrm{~ms})$. None of the interactions were significant (location transition $\times$ color transition, $\mathrm{F}(3,69)=1.172, \mathrm{p}=0.33, \eta_{\mathrm{p}}^{2}=0.05$; all other $\left.\mathrm{Fs}<1\right)$.

Planned comparisons between detection and localization procedures If there is a PRC in the standard detection procedure when the target location changes (and response repeats), then the cost for repeating the target location versus changing to the diagonal location should be weaker in the detection than in the localization procedure. This was tested with a 2 (location transition: same or change diagonal) $\times 2$ (experiment: detection or localization) mixed-factorial ANOVA, collapsing across color transition and task, as these factors did not modify the location-transition effects. There was no statistical difference between experiments on location transition, $\mathrm{F}<1$. Rather the mean estimate on the magnitude of IOR was virtually identical between the detection procedures, $t(23)=11.95, p$ $<0.01$, Mdiff $=19 \mathrm{~ms} ; 95 \% \mathrm{CI}=16-22 \mathrm{~ms}$ ) and localization procedures, $\mathrm{t}(23)=5.64, \mathrm{p}<0.01$, Mdiff $=17 \mathrm{~ms} ; 95 \% \mathrm{CI}=$ $11-24 \mathrm{~ms})$.

Recall that in the localization procedure, mean RTs on change vertical transitions were about $8 \mathrm{~ms}$ slower than the mean RTs on change horizontal transitions, a relatively small difference that was consistent with a PRC in the change vertical transition (response repeat). In the detection procedure, there was virtually no difference $(1 \mathrm{~ms})$, as expected given that both transitions theoretically resulted in PRCs. Accordingly, a 2 (location transition: change vertical or change horizontal) $\times 2$ (experiment: detection or localization) was run to test for a statistical difference between these procedures. The statistical support for this interaction was weak, unsurprisingly, given the small effect size, $\mathrm{F}(1,46)=2.83$, $\mathrm{p}=$ $0.09, \eta_{\mathrm{p}}^{2}=0.06^{5}$

\section{Discussion}

These results discredit the feature-based response hypothesis. There was no evidence that visuomotor repetition effects generally or PRCs more specifically could be generated or amplified by increasing the feature processing demands. The localization procedures produced weak PRCs (change vertical vs. change horizontal location transition), regardless of the feature-processing demands. The detection procedures provided no evidence for a visuomotor effect, as the magnitude of the cost for repeating the target location versus changing it to the diagonal in these procedures was virtually identical to the magnitude of this cost in the localization procedures.

The weakness of the PRCs in the localization procedures comes as a bit of a surprise in light of some of our recent work, 
as does the rejection of this feature-based response hypothesis in general. For example, Hilchey, Pratt, and Lamy (2019) revealed PRCs (change vertical vs. change horizontal) that were about twice as large $(\sim 15 \mathrm{~ms})$ using localization procedures that were quite similar to those used here, except the target was a color singleton in an array of three homogenously colored visual distractors (i.e., non-spatial processing was required to distinguish the targets from distractors). The magnitudes of the PRCs observed by Hilchey et al. almost tripled to about $41 \mathrm{~ms}$ when it became necessary to perform a subtle discrimination of the target shape prior to making the left/right keypress localization responses (because responses had to be withheld when the target took on a particular shape, and thus additional feature-based processing was required). On the basis of results such as these, it was tempting to predict that increasing the feature-processing demands could increase the magnitude of the PRCs, but this prediction is clearly not borne out by the current findings.

The apparent contradiction can be resolved by assuming that longer RTs are more conducive to visuomotor effects because it takes some amount of time prior to responding for the recent location-response code to be retrieved. This has been suggested to us by some researchers (Christian Frings and Roland Pfister; personal communications). Consistent with this longer RT or racehorse hypothesis, the mean RTs in Hilchey, Pratt, and Lamy's (2019) visual search task without the go/no-go requirement were about $450-550 \mathrm{~ms}$. These mean RTs increased to about $625-725 \mathrm{~ms}$ when the go/nogo shape-discrimination component was added. With the current procedures, the mean RTs were consistently below 400 ms. However, several pieces of evidence make this simple explanation unlikely. Wilson, Castel, and Pratt (2006) provided no evidence for IOR in the target-target sequences of a single keypress detection procedure with a color-based go/ no-go component, which was by design quite similar to one of our procedures but with a different result. As in our procedure, the mean RTs in Wilson et al.'s procedures were below $400 \mathrm{~ms}$. Further, vincentization approaches to examining PRCs involving location and response repetition have provided no evidence that these effects are obligatory on the slower side of the RT distribution (Huffman et al., in press). It is unlikely that a mere slowing of RT, for whatever reason, is the full story.

With little evidence to support the feature-based response hypothesis, we looked elsewhere for a possible factor that might explain why PRCs are sometimes present and sometimes not. First, many of the situations in which PRCs occur are those where two spatially separated keys are used to collect responses. In these situations, Hommel's common-coding principle $(2007,2019)$ predicts that location as a whole (i.e., of stimuli and response) is task-relevant. However, this does not account for a previous demonstration of location-based PRCs in a color-based go/no-go task with detection responses (Wilson,
Castel, \& Pratt, 2006). Upon closer reading of Wilson et al., we realized that increased spatial processing could be useful in that task for determining whether and when to respond. This is because there was a spatial contingency, such that go and no-go stimuli alternated predictably on one of the response axes. Furthermore, in Experiment 1, we only found PRCs in our localization task. Combining these insights, we designed Experiment 2 to test the prediction that it is the relevance of space to responding that determines the PRCs. We refer to this as the space-based response hypothesis

\section{Experiment 2}

The easiest way to test the space-based response hypothesis is to intermix detection and localization responses within the same target-target procedure used in Experiment 1 while making spatial processing mandatory. This is accomplished by making the response type (detection or localization) conditional on stimulus location. This approach ensures that all responses - even detection responses - are spatially defined, with the incidental effect of increasing competition at the level of response selection because of the added response option.

In Experiment 2's procedures, targets appeared at four marked locations that collectively formed an imaginary diamond centered on fixation. The responses that were required were determined by the axis on which the targets appeared. In one procedure, if the target appeared on the vertical axis (i.e., above or below fixation), it had to be localized by either an up or down arrow keypress response whereas if the target appeared on the horizontal axis (i.e., to the left or right of fixation), it had to be detected with a single ('b') keypress response. In another procedure, these mappings were reversed, such that targets on the horizontal axis had to be localized with left and right arrow keypress responses whereas targets on the vertical axis had to be detected with a single keypress response.

Our basic procedures depart purposefully from Wilson et al.'s (2006) procedures. In their procedures, it was useful to pay attention to the spatial axis upon which the target appeared (because axis roughly predicted whether and when a response would be required), but it was not strictly necessary. In our procedures, it was necessary to attend to the spatial axis in order to form the correct response. Our procedure removes Wilson et al.'s (2006) degree of spatial processing freedom (the reasoning is: why bother leaving it to the participants to learn either implicitly or explicitly that increased spatial processing is helpful for determining a response?).

If it is the case that PRCs are caused fundamentally by the relevance of spatial information to choosing between responses, then the cost for repeating the target location versus switching it to the mirror opposite location should be either nullified or reversed to a benefit with the 
detection responses. It is important to remember that this is because a PRC should theoretically occur when the target location changes (and the response repeats) but not repeats (and the response also repeats), which allows for a PRC to counteract the repetition costs driven by IOR. In contrast, the localization responses should consistently show a cost for repeating versus switching the target location. This is because a PRC should not theoretically occur when the target location repeats (and the response repeats) or the target location changes (and the response changes). Put differently, there is no opponent process in the TEC that can theoretically overshadow IOR with localization responses when there are 1:1 correspondences between stimulus locations and responses. We also ran a control procedure, which was identical in every way except that only left-handed keypress detection responses were required. This was run mainly to ensure that our placeholder layout changes (imaginary square in E1; imaginary diamond in E2) would not somehow be responsible for attenuating IOR with detection responses. It was also included because we needed a baseline procedure that involved left-handed keypress detection responses, which is how detection was made in the intermixed localization and detection procedures (detection responses were made with the right hand in Experiment 1).

\section{Method}

Participants Forty-eight undergraduates from the University of Toronto consented to participate for course credit ( $\mathrm{M}$ age $=18.81$ years; two left-handed; 11 males).

Stimuli and apparatus These were kept as similar as possible to the standard procedures in Experiment 1, with two exceptions: (1) The peripheral placeholder squares were arranged to form an imaginary diamond, instead of an imaginary square, centered on fixation, and (2) the peripheral placeholders randomly turned red or green, but not blue, as a third color was unnecessary to test the space-based attention hypothesis and we were still able to analyze effects of color transition without it.

Procedure and design The timing of stimulus events was identical to Experiment 1. Each trial began with the placeholder array, followed by the onset of the fixation stimulus, the first colored stimulus at a random placeholder location in peripheral vision, the white circle at fixation, and then the second colored stimulus at a random placeholder location in peripheral vision. The trial ended with an instruction screen if an error was made and a blank 1-s inter-trial interval regardless of error.

Forty-eight participants were divided evenly among the three procedures, which differed on the response rules to the targets. In the "localize horizontal, detect vertical" procedure, left arrow (right index finger) and right arrow (right middle finger) keypress responses were required to stimuli appearing in the left and right placeholders, respectively, whereas a ' $b$ ' keypress (left index finger) response was required to stimuli appearing in the top and bottom placeholders. In the "detect horizontal, localize vertical" procedure, up arrow (right middle finger) and down arrow (right index finger) keypress responses were required to stimuli appearing in the top and bottom placeholders, respectively, whereas a 'b' keypress (left index finger) response was required to stimuli appearing in the left and right placeholders. In both of these procedures, all three keys had to be pressed at the same time in response to the white circle at fixation. In the standard detection procedure, $a$ ' $b$ ' keypress response (left index finger) was required to each stimulus. All such stimuli remained onscreen until a response was made. The "localize horizontal, detect vertical" procedure is illustrated by Fig. 4.

In the "localize horizontal, detect vertical" and "detect horizontal, localize vertical" procedures, participants completed 20 practice trials, followed by 512 experimental trials, within which the color and location of the peripheral targets randomly repeated. In the standard detection procedure, participants completed 20 practice trials, followed by 512 experimental trials, within which the color and location of the peripheral targets randomly repeated. Unique to the standard detection procedure, there were an additional 64 "catch" trials in which the second peripheral target was omitted to help minimize anticipatory responses.

\section{Results}

Standard detection procedure (control experiment) The mean false-alarm rate was 6.5\% (range $=0.0-18.8 \%$; $\mathrm{SD}=5.3$ ). Participants failed to respond to either the first or second peripheral stimulus on $0.3 \%$ of trials, which were excluded. An additional two trials were excluded for exceptionally slow responses ( $>3 \mathrm{~s}$ ). For ease of comparison with the other two procedures, half of the data were excluded because the target's axis changed between the first and second peripheral targets, which constituted a task switch in the other two procedures. All trials with RTs to the second peripheral target less and greater than 3 standard deviations from the participant's mean were excluded, resulting in the loss of $1.5 \%$ of the remaining data.

Effects of repeating/changing the locations and colors of peripheral targets on RTs were analyzed with a 2 (location transition: repeat or change) $\times 2$ (color transition: repeat or change) $\times 2$ (axis: horizontal or vertical) repeated-measures ANOVA. Figure 5 illustrates the mean RTs across all levels of the experiment.

The main effect of location transition was significant, $\mathrm{F}(1$, $15)=46.35, p<0.01, \eta_{p}^{2}=0.76$, with slower responses when the target location repeated $(338 \mathrm{~ms})$ instead of switched $(320$ 


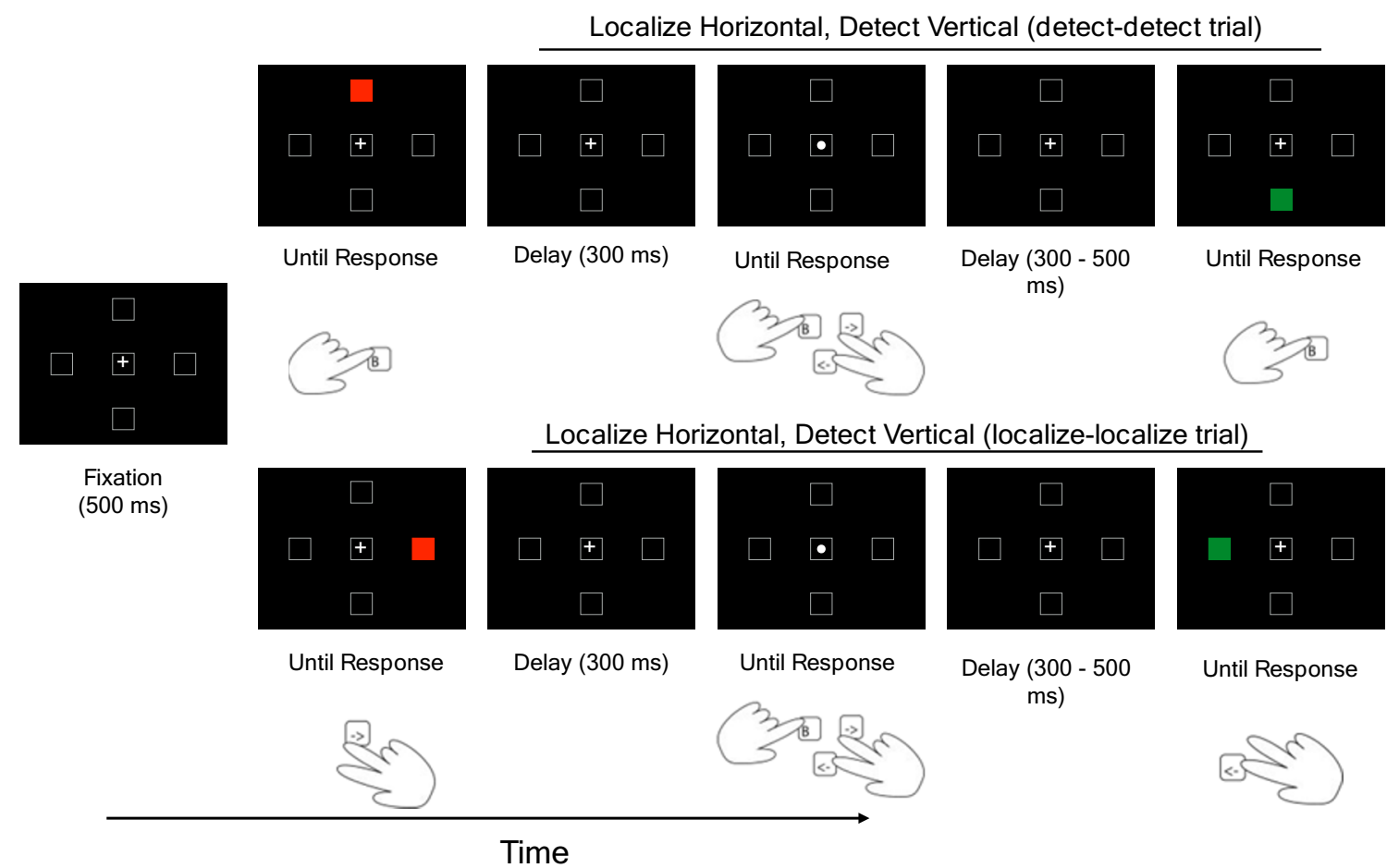

Fig. 4 Two of the possible trial sequences are illustrated for the "Localize Horizontal, Detect Vertical" procedure. Colored squares that appear to the left and right of the fixation point are responded to by the left and right arrow keys, respectively, whereas those that appear above and below fixation are detected with a single button press. As per usual, the target

$\mathrm{ms}$ ), consistent with IOR, but there were no other significant effects or interactions (location transition $\times$ color transition, $F(1,15)=2.23, p=0.16, \eta_{p}^{2}=0.13$; all other $\left.F s<1\right)$.

Localize horizontal, detect vertical procedure We excluded $0.5 \%$ of the data for exceptionally slow responses (> $3 \mathrm{~s}$ ). Trials with response errors to the first peripheral target (1.8\%) were excluded from the subsequent error rate and RT analysis whereas trials with errors to the second peripheral target $(3.4 \%)$ were only excluded from the RT analysis. Half of the data were excluded because the peripheral target's axis changed (i.e., the first peripheral target was on the vertical axis and the second was on the horizontal or vice versa), which constituted a task switch. For the RT analysis, all trials with RTs to the second peripheral target less or greater than 3 standard deviations from the participant's mean detection (vertical axis) and localization (horizontal axis) RT were excluded, resulting in the loss of $1.9 \%$ of the remaining data.

Effects of repeating/changing the locations and colors of peripheral targets on RTs were analyzed with a 2 (location transition: repeat or change) $\times 2$ (color transition: repeat or change) $\times 2$ (axis: horizontal or vertical) repeated-measures ANOVA. Figure 6 illustrates the mean RTs across all levels of the experiment.

Neither the main effects of location transition, $F(1,15)=$ 2.997, $\mathrm{p}=0.10, \eta_{\mathrm{p}}^{2}=0.17$, nor color repetition, $\mathrm{F}<1$, were color and location randomly repeats. In the above example, both colored squares appeared on the vertical axis and thus had to be localized. In the bottom example, both colored squares appeared on the horizontal axis and thus had to be localized. In both examples, the target color and location changed

significant. There was a main effect of axis, $F(1,15)=51.02$, $p$ $<0.01, \eta_{p}^{2}=0.77$, with localization responses to targets on the horizontal axis being much faster than detection responses to targets on the vertical axis (418 vs. $519 \mathrm{~ms}$ ).

The interaction between location transition and axis was significant, $F(1,15)=13.15, p<0.01, \eta_{p}^{2}=0.47$. For the horizontal axis, a planned comparison indicated that responses were slower when the target location repeated $(430 \mathrm{~ms})$ instead of switched $(406 \mathrm{~ms} ; \mathrm{t}(15)=3.65, \mathrm{p}<0.01$, Mdiff $=24$ $\mathrm{ms} ; 95 \% \mathrm{CI}=10-38 \mathrm{~ms}$ ), consistent with IOR. For the vertical axis, a planned comparison failed to reveal a difference between repeating the target location $(517 \mathrm{~ms})$ and switching it $(522 \mathrm{~ms} ; \mathrm{t}(15)=-0.67, \mathrm{p}=0.51, \mathrm{Mdiff}=-5 \mathrm{~ms} ; 95 \% \mathrm{CI}=-20$ $10 \mathrm{~ms}$ ), consistent with a PRC in addition to IOR.

Unexpectedly, the interaction between axis and color transition approached significance, $F(1,15)=3.46, p=0.08, \eta^{2}{ }_{p}=$ 0.19 . For localization responses on the horizontal axis, responses were slightly faster when the target color repeated (413 ms) instead of switched (423 ms), with an unplanned comparison showing that this effect was indeed significant, $\mathrm{t}(15)=-2.36, \mathrm{p}=0.03$, Mdiff $=-10 \mathrm{~ms} ; 95 \% \mathrm{CI}=-19-(-1)$ $\mathrm{ms})$. For detection responses on the vertical axis, responses were slightly slower when the target color repeated $(521 \mathrm{~ms})$ instead of switched $(518 \mathrm{~ms})$, but not statistically so $(\mathrm{t}(15)=$ $0.63, \mathrm{p}=0.53$, Mdiff $=3 \mathrm{~ms} ; 95 \% \mathrm{CI}=-9-17 \mathrm{~ms})$. The remaining interactions were not significant $(\mathrm{Fs}<1)$. 


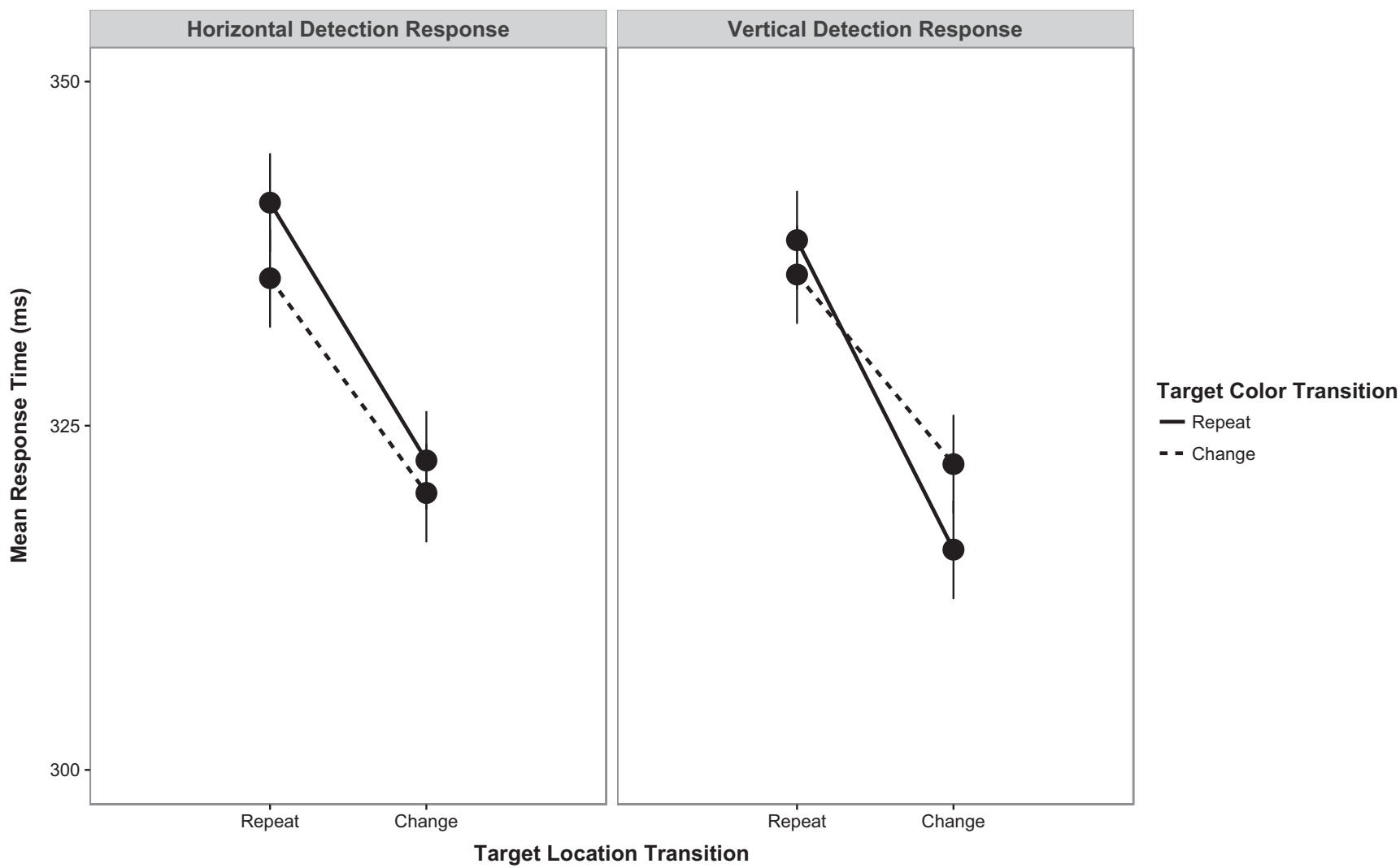

Fig. 5 Mean response times for all combinations of target location transition, target color transition, and response axis (columns) in the detection procedure in Experiment 2. Error bars are half Fisher's Least
Significant Difference (FLSDs) computed from the mean squared error term of the interaction

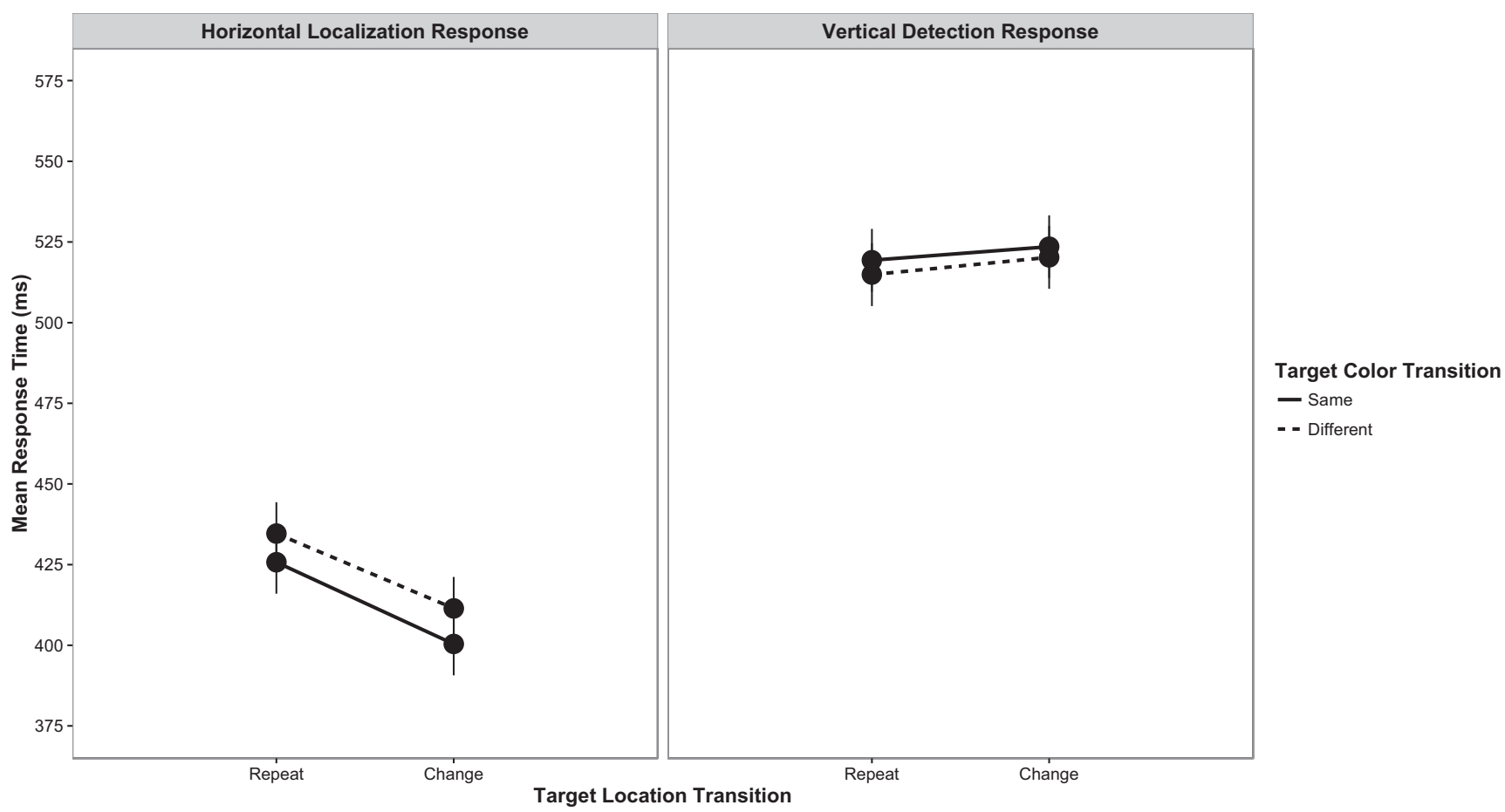

Fig. 6 Mean response times for all combinations of target location transition, target color transition, and response axis (columns) in the "localize horizontal, detect vertical" procedure in Experiment 2. Error bars are half Fisher's Least Significant Difference (FLSDs) computed from the mean squared error term of the interaction 
Keypress errors to the appearance of the targets were also analyzed. The main effect of location transition was significant, $\mathrm{F}(1,15)=4.95, \mathrm{p}=0.04, \eta_{\mathrm{p}}^{2}=0.25$, with more errors for target location repeats $(4.4 \%)$ than switches $(3.1 \%)$. The main effect of axis was also significant, with more errors for localization responses on the horizontal axis $(5.3 \%)$ than detection responses on the vertical axis $(2.2 \%)$. Thus, when considered with the RT analysis, the main effect of axis at least partly reflected a speed-accuracy tradeoff. No other effects or interactions approached significance (all other ps $>0.16$ ).

Detect horizontal, localize vertical procedure We excluded $1.4 \%$ of the data for exceptionally slow responses $(>3 \mathrm{~s})$. Trials with response errors to the first peripheral target (2.5\%) were excluded from the subsequent error rate and RT analyses whereas trials with errors to the second peripheral target (3.7\%) were only excluded from the RT analyses. Half of the data were excluded because the peripheral target's axis changed. All trials with RTs to the second peripheral target less or greater than 3 standard deviations from the participant's mean detection (horizontal axis) and localization (vertical axis) RTs were excluded, resulting in the loss of $2.1 \%$ of the remaining data.

Effects of repeating/changing the locations and colors of peripheral targets on the RTs were analyzed with a 2 (location transition: repeat or change) $\times 2$ (color transition: repeat or change) $\times 2$ (axis: horizontal or vertical) repeated-measures ANOVA. Figure 7 illustrates the mean RTs across all levels of the experiment. Neither the main effects of location transition, $\mathrm{F}<1$, nor color transition, $\mathrm{F}(1,15)=2.13, \mathrm{p}=0.17, \eta_{\mathrm{p}}^{2}=0.12$, were significant. There was again a main effect of axis, $\mathrm{F}(1$, $15)=31.38, p<0.01, \eta_{p}^{2}=0.68$, with much faster localization responses to targets on the vertical axis $(431 \mathrm{~ms})$ than detection responses to targets on the horizontal axis $(518 \mathrm{~ms})$.

The interaction between location transition and axis was significant, $F(1,15)=22.67, p<0.01, \eta_{p}^{2}=0.60$. For the horizontal axis, a planned comparison revealed that detection responses were faster when the target location repeated (505 $\mathrm{ms}$ ) instead of switched (530 ms; $\mathrm{t}(15)=-2.54, \mathrm{p}=0.02$, Mdiff $=-25 \mathrm{~ms}, 95 \% \mathrm{CI}=-45-(-4) \mathrm{ms})$, consistent with a PRC. For the vertical axis, a planned comparison revealed that localization responses were slower when the target location repeated $(449 \mathrm{~ms})$ instead of switched (414 ms; $\mathrm{t}(15)=4.85, \mathrm{p}<0.01$, Mdiff $=35 \mathrm{~ms} ; 95 \% \mathrm{CI}=20-50 \mathrm{~ms}$ ), consistent with IOR.

Yet again, there appeared to be a relationship between color repetition and axis, $\mathrm{F}(1,15)=6.53, \mathrm{p}=0.02, \eta_{\mathrm{p}}^{2}=0.30$. Detection RTs to targets on the horizontal axis were faster when the target color repeated $(508 \mathrm{~ms})$ instead of switched $(527 \mathrm{~ms} ; \mathrm{t}(15)=-2.16, \mathrm{p}=0.04, \operatorname{Mdiff}=19 \mathrm{~ms} ; 95 \% \mathrm{CI}=-$ $38-(-1) \mathrm{ms})$. Localization RTs to targets on the vertical axis were similar regardless of whether the target color repeated $(433 \mathrm{~ms})$ or switched $(429 \mathrm{~ms} ; \mathrm{t}(15)=0.96, \mathrm{p}=0.35$, Mdiff $=$ $4 \mathrm{~ms} ; 95 \% \mathrm{CI}=-4-12 \mathrm{~ms})$. The remaining interactions were not significant $(\mathrm{Fs}<1)$.
Error rate analyses revealed no main effects or interactions (all Fs $<1$ ).

\section{Discussion}

The results of the second experiment were unequivocally consistent with the space-based response hypothesis. When only detection responses were made (i.e., the control procedure), responses were slower when the target location repeated instead of switched the mirror opposite location, consistent with an IOR effect and the results of Experiment 1. When spacebased processing was needed to decide between making localization or detection responses, detection RTs changed in a way that indicated that a visuomotor repetition effect was occurring. Specifically, detection RTs were either unaffected by target location repetition (the localize horizontal, detect vertical procedure), consistent with Wilson et al. (2006), or made faster by target location repetition (localize vertical, detect horizontal procedure). The localization responses were consistently slower when the target location repeated. Collectively, the results from the non-standard procedures are consistent with the TEC, as only detection responses could theoretically incur a PRC (i.e., when the target location changed and response repeated), which would overshadow IOR. Keeping within the TEC framework, the results suggest that PRCs at the level of location and response repetition are especially likely to come into play when increased spatial processing occurs to guide response selection.

Unexpectedly, even though feature-based processing was not required in order to produce the correct responses in these procedures, both the detect horizontal, localize vertical, and localize horizontal, detect vertical procedures provided some evidence that repeating versus switching the target color led to faster RTs on the horizontal but not vertical axis. There was no evidence for this in the control procedure. The reasons for these relative color repetition benefits on the horizontal axis are unclear but they must have something to do with the added response selection demands. For now, we note simply that, as per usual with distractor-less displays, target color repetition did not modify the target location repetition effects (e.g., Huffman et al., 2018; Hilchey, Rajsic, et al., 2018) and that, across all procedures and experiments, any RT advantage for repeating a target color was quite weak, with the lower bounds on the $95 \%$ confidence intervals always approaching $0 \mathrm{~ms}$.

\section{General discussion}

The starting point for this investigation was the simple observation that in some variations of the well-known spatial cueing paradigm, repeating a stimulus location leads to a pattern of benefits and costs that is response-dependent, producing visuomotor effects consistent with the PRCs predicted by 


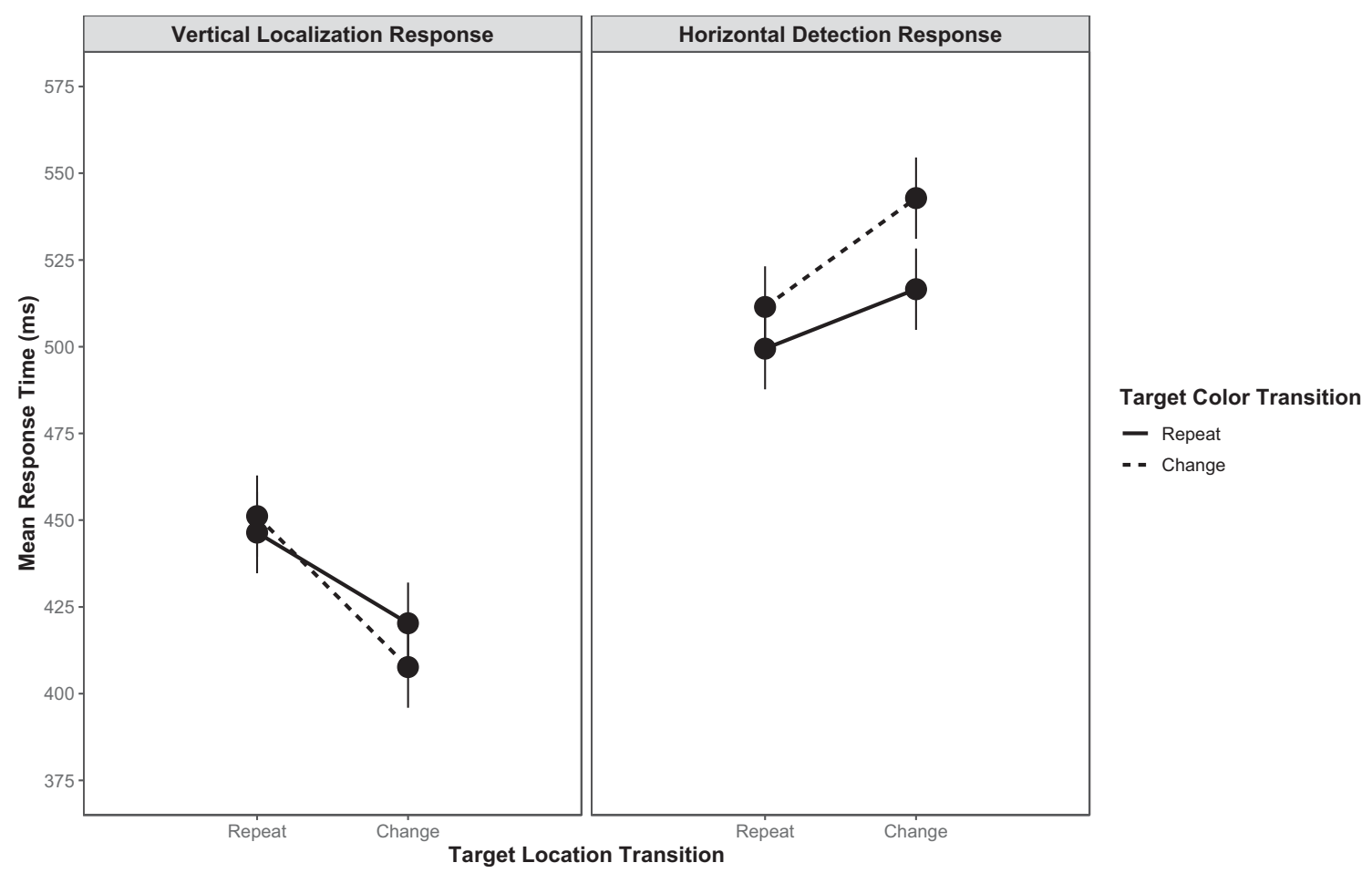

Fig. 7 Mean response times for all combinations of target location transition, target color transition, and response axis (columns) in the "detect horizontal, localize vertical" procedure in Experiment 2. Error bars are half Fisher's Least Significant Difference (FLSDs) computed from the mean squared error term of the interaction the TEC framework. Other variations lead to no such response-dependence, producing instead stimulus location repetition costs consistent with IOR as predicted by attentional orienting and visuospatial selection frameworks. The present experiments were designed to test whether differences in how much feature- or space-based processing can explain when visuomotor repetition effects are likely to occur and, by extension, why stimulus location repetitions sometimes reveal PRCs, while at other times IOR.

Experiment 1 tested the feature-based response hypothesis, which stipulates that visuomotor effects are caused fundamentally by any increase in feature-based processing. We tested this by manipulating, within target-target keypress detection procedures with 4:1 location-response mappings and keypress localization procedures with 2:1 location-response mappings, whether it was necessary to process color information in order to make the appropriate response. The results within and across these procedures were clear-cut. Within the localization procedures, responses were slightly slower when the target location switched but the response repeated relative to when the target location switched and the response switched, with both locations being equi-distant from the last target location. This result is consistent with a PRC. This procedure also revealed that responses were slower when the target location and response repeated instead of switched to the mirror opposite location. This result is consistent with IOR. The detection procedure revealed that responding was slower when the target location repeated relative to switched, which is also consistent with IOR. Most important, there was no evidence within these procedures that these effects depended on feature-based processing, as these effects were stable regardless of whether stimulus color processing was needed in order to determine response execution. Further, the estimate on the magnitude of IOR was virtually indistinguishable across the detection and localization procedures, which would not be the case if a PRC occurred in the detection procedure when the target location changed. Put simply, the detection procedure provided no evidence of a visuomotor repetition effect and the localization procedures provided weak evidence of a visuomotor repetition effect - a PRC, specifically - with no modulation by the feature-based processing demands in either case. Altogether, Experiment 1 provides virtually no support for the feature-based response hypothesis.

Experiment 2 tested the space-based response hypothesis, which stipulates that visuomotor repetition effects are caused by increases in the spatial processing that is required in order to choose a response. We tested this by intermixing single keypress detection response (2:1 location response mappings) and two-choice localization responses (1:1 location-response mappings) within the same procedure, such that targets appearing above or below fixation had to be detected whereas targets appearing to the left and right of fixation had to be localized, or vice versa. This approach ensured that (1) space was directly relevant for all responses, (2) there was 
incidentally added competition at the level of response selection (on account of the added response option(s)), and (3) only the detection responses could theoretically show PRCs, according to the TEC. The localization responses consistently showed slower responses when the target location repeated instead of switched to the mirror opposite location, consistent with IOR. However, this cost was significantly diminished for the detection responses, even reversing to a benefit in one case. The pattern is consistent with a PRC in the detection procedure at the location opposite the previous target, whose magnitude could become equal to or greater than any cost of repeating the target location. A control condition demonstrated that the detection responses showed the expected cost for repeating the target location when the need to process spatial information was eliminated by removing the localization responses from the procedure. In short, Experiment 2 provides strong support for the space-based response hypothesis.

There are nuances that need to be considered in the interpretation of these results. First, the results of these experiments should not be taken to discredit the role of feature-based processing for the retrieval of all event representations. Interactions between response and stimulus location repetition represent a single instance of a class of visuomotor repetition effects, which belongs to an even broader class of integration effects (Hommel \& Colzato, 2009). The visuomotor class includes interactions between stimulus color and response location, stimulus shape and response location, and stimulus category and response location, all of which are dissociable, and some of which are known to be modified by the extent to which feature-based processing is required (Hommel, Memelink, Zmigrod, \& Colzato, 2014; Huffman et al., in press; Moeller \& Frings, 2014; Singh, Moeller, Koch, \& Frings, 2018). Rather, the results from the experiments here suggest specifically that feature-based processing does not directly or obligatorily modify visuomotor repetition effects at the level of stimulus location and response repetition. In addition, our results also do not attest to the role of attention, or selective processing, in retrieving event files that do not include a response component (e.g., color $\times$ shape interactions; see Hommel, 1998, for examples).

Second, the experiments leave open the general possibility that event file retrieval effects are a by-product of the type of attention (e.g., color-based, space-based, shape-based, category-based, etc.) that is deemed suitable for planning a response (Henson, Eckstein, Waszak, Frings, \& Horner, 2014; Hommel, 1998; Memelink \& Hommel, 2013; Schumacher \& Hazeltine, 2016). In this respect, it is noteworthy in Experiment 1 that color processing determined whether to make a response but not how to make it. This is different than typical two-alternative forced choice (AFC) target identification procedures, in which it is not clear how to make the response until the target is identified (e.g., Hilchey et al., 2017a; Hommel, 1998). In Experiment 2's intermixed detection and localization procedures, spatial processing directly determined how to respond, with the response depending on a successful identification of the target's spatial axis. The findings allow for the more general theory that attention, in the context of response or task sets, is fundamentally responsible for all event file retrieval effects. Determining how attention is tuned across different dimensions and what event representation will be retrieved is an outstanding issue. We speculate broadly that that all event representation retrieval may be conditional on the task-specific response processing requirements, such that subtle procedural differences that change the selection emphases will dramatically shift the levels on which these effects are observed. Whether the field wishes to unite the many selective processing differences that can occur in order to form a behavioral response under the broad umbrella of attention is something that researchers will need to work on together to resolve (Hommel et al., 2019).

Third, we wish to reiterate that the kind of visuomotor repetition effect (the PRC, more specifically) that is shown here is not related to the efficiency of target detection, the initial exogenous or endogenous shift of attention to a target location, or to visual search at all, as shown elsewhere (e.g., Hilchey, Antinucci, et al., 2019; Hilchey, Rajsic, et al., 2018; Hilchey et al., 2017b; Zehetleitner, Rangelov, \& Muller, 2012). The effects occur after an initial shift of attention toward a stimulus. Further, the effects shown here are not strictly on perception and they are not strictly on action. Specifically, the effects concern the relationship between perception and action, with selective attention conceivably playing a role in retrieving associations that have been formed between them. Experiment 2 was especially useful in this regard for showing, in particular, that increasing the spatial processing demand in order to form a response can cause visuomotor repetition effects, in line with the space-based response hypothesis.

Fourth, in light of other findings, it remains notable that target color and location repetition do not interact in targettarget procedures without visual distractors, regardless of whether keypress detection, localization (as shown here; see also Huffman et al., 2018, for review) or color discrimination responses are required (e.g., Hilchey et al., 2017a). Attention to color is simply not sufficient for retrieving event files at the level of color and location repetition. Furthermore, it remains clear that non-spatial IOR (any cost for repeating a stimulus identity; e.g., Fox \& de Fockert, 2001; Law, Pratt, \& Abrams, 1995), independent of space, does not materialize in targettarget detection and localization procedures (Kwak \& Egeth, 1992). When responses are not made to the first of two stimuli in a sequence (i.e., cue-target procedures), there is some evidence that color repetition can modify spatial priming effects, or theoretically IOR (e.g., Hu, Fan, Samuel, \& He, 2013; Klein et al., 2015). The interaction, when it occurs, is such that the magnitude of IOR can be larger when the target and 
cue colors match instead of mismatch, independent of response repetition. The results are unusual from the perspective of the TEC, insofar as the experiments that gave rise to the TEC provided little support for interactions between stimulus location and color repetition (Hommel, 1998, 2007; see also Hilchey, Pratt, \& Lamy, 2019, for additional consideration in the context of visual search). Further, even if the TEC were to allow for this interaction, the nature of it is not as expected given the partial mismatching principles. Our interpretation of the TEC is that a PRC at the level of stimulus location and color repetition would artificially increase the magnitude of IOR. This is because PRCs would theoretically occur whenever the stimulus location repeats and color switches and whenever the stimulus location switches and color repeats, the outcome of which would be lesser IOR on color repeat trials and greater IOR on color switch trials. The interaction between stimulus color and location repetition in the cuetarget procedure is consistent with habituation and cue-target integration/segregation theories (e.g., Cochrane \& Milliken (2020); Klein, Wang, Dukewich, \& Hu, 2015; Lupianez, 2010; Lupiáñez et al., 2013), but it is not consistent with the TEC.

Fifth, color priming effects (i.e., effects of repeating colors) in these experiments were weak to non-existent (though generally positive), including in the procedures of Experiment 1 in which attention to color was unambiguously necessary to respond appropriately (though not necessarily to plan the response). These findings are consistent with the literature, as it is known that the advantage for repeating the target color is usually only robust when the target is accompanied by visual distractors, such that attention to color becomes useful or indispensable for finding the target (e.g., Goolsby \& Suzuki, 2001; Hilchey, Antinucci, et al., 2019; Huffman \& Pratt, 2017; Milliken, Joordens, Merikle, \& Seiffert, 1998; Yashar, White, Fang, \& Carrasco, 2017). Given that Experiment 1 demonstrates that attention to the target color after it has been found does not result in robust color priming effects, our suspicion is that attention to color generally is not sufficient for color priming. Rather, in line with other researchers, we suspect that it is a particular type of attention that is necessary to produce color priming effects in visual search. It is specifically the type of attention that must be allocated to color in order to locate the target. The color priming phenomenon is thus fundamentally search-related, putting aside at what levels, exactly, the effects are taking place (Allenmark et al., 2018; Burnham, 2018).

Finally, we wish to reinforce that our results speak directly to the cause of visuomotor repetition effects regardless of whether or not they are interpreted as PRCs through the lens of the TEC. Visuomotor repetition effects do not need to be defined strictly in terms of the interference that may arise when there is a mismatch between a retrieved event representations and the current processing event, even if many findings, including those presented here, are consistent with this perspective (Hommel \& Colzato, 2009). Looking at some of our results from a different angle, an alternative interpretation of Experiment 2 could stress that IOR may not have been counteracted by a PRC at all. Rather, for the detection but not localization responses, it could be argued that IOR was counteracted by a reactive tendency to reproduce the recently associated response when the stimulus location repeated. The findings follow logically from Pashler and Baylis' (1991) notions of a stimulus-response repetition shortcut. In that theory, a stimulus-response repetition shortcut, or heuristic, is most likely when response selection is difficult. The data in the intermixed localization and detection procedures are consistent with this proposal, in showing that detection responses were much slower than localization responses when intermixed whereas detection responses were relatively fast when the localization responses were removed from the procedure. The relative slowing of the detection responses in the intermixed conditions presumably arises because these responses were not directly compatible with any of the target locations in the context of the competition for response selection, whereas all localization responses were (see, e.g., Proctor $\& \mathrm{Vu}, 2006$, for review of S-R compatibility). Thus, the conditions were theoretically favorable for a stimulus locationresponse repetition heuristic with the detection responses specifically, in large part because these responses were disproportionately difficult to choose. The difference between these frameworks is whether IOR was masked in the detection procedure because of a cost for switching the target location (i.e., the PRC) or because of a benefit for repeating the target location (i.e., a stimulus location-response repetition heuristic). These frameworks need not be mutually exclusive, and regardless of whichever is preferred, the findings make clear that event representation retrieval becomes most likely whenever a particular dimension may be processed more deeply in order to determine a response.

To summarize, the goal of the study was to identify factors that determine when a spatial cueing procedure is more likely to reveal visuomotor than visuospatial repetition effects, through the lens of the TEC. Our first study demonstrated that differences in the feature-based processing demands do not directly determine visuomotor repetition effects involving stimulus location and response repetition, inconsistent with the feature-based response hypothesis. Our second study demonstrated that increased spatial-processing demands for the response directly determine visuomotor repetition effects involving stimulus location and response repetition, consistent with the space-based response hypothesis. The findings compel theories to acknowledge that any situation that allows for any choice amongst response options to be aided by increased spatial processing is likely to result in visuomotor repetition effects at the level of stimulus location and response repetition, any other effects of or on attention notwithstanding. As 
this precondition is met by many studies exploring how visual selective attention is biased by recently processed stimulus locations, many studies understandably provide evidence of visuomotor repetition effects, among other things.

Open Practices Statement The aggregate RT data for the experiments reported here are available at https:/osf.io/ud7y2/ (https://doi.org/10.17605/OSF.IO/UD7Y2). Additional information is available upon request. None of these experiments were preregistered.

\section{References}

Adam, J. J., \& Pratt, J. (2004). Dissociating visual attention and effector selection in spatial precuing tasks. Journal of Experimental Psychology: Human Perception and Performance, 30(6), 10921106.

Allenmark, F., Müller, H. J., \& Shi, Z. (2018). Inter-trial effects in visual pop-out search: Factorial comparison of Bayesian updating models. PLoS computational biology, 14(7), e1006328.

Burnham, B. R. (2018). Selection and response bias as determinants of priming of pop-out search: Revelations from diffusion modeling. Psychonomic bulletin \& review, 25(6), 2389-2397.

Carrasco, M. (2011). Visual attention: The past 25 years. Vision research, 51(13), 1484-1525.

Chica, A. B., Martín-Arévalo, E., Botta, F., \& Lupiánez, J. (2014). The Spatial Orienting paradigm: How to design and interpret spatial attention experiments. Neuroscience \& Biobehavioral Reviews, 40, 35-51.

Christie, J., \& Klein, R. M. (2001). Negative priming for spatial location?. Canadian Journal of Experimental Psychology/Revue canadienne de psychologie expérimentale, 55(1), 24-38.

Christie, J. J., \& Klein, R. M. (2008). On finding negative priming from distractors. Psychonomic bulletin \& review, 15(4), 866-873.

Cochrane, B. A., \& Milliken, B. (2020). The representational basis of positive and negative repetition effects. Journal of Experimental Psychology: Human Perception and Performance, 46(3), 252-263.

Coward, R. S., Poliakoff, E., O'Boyle, D. J., \& Lowe, C. (2004). The contribution of non-ocular response inhibition to visual inhibition of return. Experimental brain research, 155(1), 124-128.

D’Angelo, M. C., Thomson, D. R., Tipper, S. P., \& Milliken, B. (2016). Negative priming 1985 to 2015: A measure of inhibition, the emergence of alternative accounts, and the multiple process challenge. The Quarterly Journal of Experimental Psychology, 69(10), 18901909.

Dukewich, K. R. (2009). Reconceptualizing inhibition of return as. Psychonomic bulletin \& review, 16(2), 238-251.

Eng, V., Lim, A., Kwon, S., Gan, S. R., Jamaluddin, S. A., Janssen, S. M., \& Satel, J. (2017). Stimulus-response incompatibility eliminates inhibitory cueing effects with saccadic but not manual responses. Attention, Perception, \& Psychophysics, 79(4), 1097-1106.

Fox, E., \& de Fockert, J. W. (2001). Inhibitory effects of repeating color and shape: Inhibition of return or repetition blindness?. Journal of Experimental Psychology: Human Perception and Performance, 27(4), 798-812.

Frings, C., Schneider, K. K., \& Fox, E. (2015). The negative priming paradigm: An update and implications for selective attention. Psychonomic bulletin \& review, 22(6), 1577-1597.
Goolsby, B. A., \& Suzuki, S. (2001). Understanding priming of colorsingleton search: Roles of attention at encoding and "retrieval". Perception \& Psychophysics, 63(6), 929-944.

Guy, S., Buckolz, E., \& Pratt, J. (2004). The influence of distractor-only prime trials on the location negative priming mechanism. Experimental Psychology, 51(1), 4-14.

Henson, R. N., Eckstein, D., Waszak, F., Frings, C., \& Horner, A. J. (2014). Stimulus-response bindings in priming. Trends in cognitive sciences, 18(7), 376-384.

Hilchey, M. D., Antinucci, V., Lamy, D., \& Pratt, J. (2019). Is attention really biased toward the last target location in visual search? Attention, response rules, distractors, and eye movements. Psychonomic bulletin \& review, 26(2), 506-514.

Hilchey, M. D., Klein, R. M., \& Satel, J. (2014). Returning to "inhibition of return" by dissociating long-term oculomotor IOR from shortterm sensory adaptation and other nonoculomotor "inhibitory" cueing effects. Journal of Experimental Psychology: Human Perception and Performance, 40(4), 1603-1616.

Hilchey, M. D., Leber, A. B., \& Pratt, J. (2018). Testing the role of response repetition in spatial priming in visual search. Attention, Perception, \& Psychophysics, 80(6), 1362-1374.

Hilchey, M. D., Pratt, J., \& Lamy, D. (2019). Is attention really biased toward the last target location in visual search? The role of focal attention and stimulus-response translation rules. Journal of experimental psychology: human perception and performance, 45(10), 1415-1428

Hilchey, M. D., Rajsic, J., Huffman, G., Klein, R. M., \& Pratt, J. (2018). Dissociating orienting biases from integration effects with eye movements. Psychological science, 29(3), 328-339

Hilchey, M. D., Rajsic, J., Huffman, G., \& Pratt, J. (2017a). Intervening response events between identification targets do not always turn repetition benefits into repetition costs. Attention, Perception, \& Psychophysics, 79(3), 807-819.

Hilchey, M. D., Rajsic, J., Huffman, G., \& Pratt, J. (2017b). Responsemediated spatial priming despite perfectly valid target location cues and intervening response events. Visual Cognition, 25(9-10), 888902.

Hommel, B. (1998). Event files: Evidence for automatic integration of stimulus-response episodes. Visual Cognition, 5(1-2), 183-216.

Hommel, B. (2004). Event files: Feature binding in and across perception and action. Trends in cognitive sciences, 8(11), 494-500.

Hommel, B. (2005). How much attention does an event file need? Journal of Experimental Psychology: Human Perception and Performance, 31(5), 1067-1082.

Hommel, B. (2007). Feature integration across perception and action: Event files affect response choice. Psychological research, 71(1), 42-63.

Hommel, B. (2019). Theory of Event Coding (TEC) V2. 0: Representing and controlling perception and action. Attention, Perception, \& Psychophysics, 1-16.

Hommel, B., Chapman, C. S., Cisek, P., Neyedli, H. F., Song, J. H., \& Welsh, T. N. (2019). No one knows what attention is. Attention, Perception, \& Psychophysics, 81(7), 2288-2303.

Hommel, B., \& Colzato, L. S. (2009). When an object is more than a binding of its features: Evidence for two mechanisms of visual feature integration. Visual Cognition, 17(1-2), 120-140.

Hommel, B., Memelink, J., Zmigrod, S., \& Colzato, L. S. (2014). Attentional control of the creation and retrieval of stimulusresponse bindings. Psychological research, 78(4), 520-538.

Hommel, B., Müsseler, J., Aschersleben, G., \& Prinz, W. (2001). The theory of event coding (TEC): A framework for perception and action planning. Behavioral and brain sciences, 24(5), 849-878.

Hommel, B., Proctor, R. W., \& Vu, K. P. L. (2004). A feature-integration account of sequential effects in the Simon task. Psychological research, 68(1), 1-17. 
Hommel, B., \& Wiers, R. W. (2017). Towards a unitary approach to human action control. Trends in Cognitive Sciences, 21(12), 940949.

Hu, F. K., Fan, Z., Samuel, A. G., \& He, S. (2013). Effects of display complexity on location and feature inhibition. Attention, Perception, \& Psychophysics, 75(8), 1619-1632.

Huffman, G., Hilchey, M. D., \& Pratt, J. (2018). Feature integration in basic detection and localization tasks: Insights from the attentional orienting literature. Attention, Perception, \& Psychophysics, 80(6), 1333-1341.

Huffman, G., Hilchey, M. D., Weidler, B. J., Mills, M., \& Pratt, J. (2020). Does feature-based attention play a role in the episodic retrieval of event files. Journal of Experimental Psychology: Human Perception \& Performance.

Huffman, G., \& Pratt, J. (2017). The action effect: Support for the biased competition hypothesis. Attention, Perception, \& Psychophysics, 79(6), 1804-1815.

Ivanoff, J., \& Klein, R. M. (2004). Stimulus-response probability and inhibition of return. Psychonomic Bulletin \& Review, 11(3), 542550 .

Kingstone, A. (1992). Combining expectancies. The Quarterly Journal of Experimental Psychology Section A, 44(1), 69-104.

Kingstone, A., \& Pratt, J. (1999). Inhibition of return is composed of attentional and oculomotor processes. Perception \& Psychophysics, 61(6), 1046-1054.

Klein, R. (2009). On the control of attention. Canadian Journal of Experimental Psychology/Revue canadienne de psychologie expérimentale, 63(3), 240-252.

Klein, R. M. (2000). Inhibition of return. Trends in cognitive sciences, 4(4), 138-147.

Klein, R. M. (2004). Orienting and inhibition of return. In M. S. Gazzaniga (Ed.), The cognitive neurosciences (pp. 545-559). Cambridge, MA: MIT Press.

Klein, R. M., \& Redden, R. S. (2018). Two "inhibitions of return" bias orienting differently. In T. L. Hubbard (Ed.), Spatial biases in perception and cognition (pp. 295-306). New York, NY: Cambridge University.

Klein, R. M., Wang, Y., Dukewich, K. R., He, S., \& Hu, K. (2015). On the costs and benefits of repeating a nonspatial feature in an exogenous spatial cuing paradigm. Attention, Perception, \& Psychophysics, 77(7), 2293-2304.

Koch, I., Frings, C., \& Schuch, S. (2018). Explaining response-repetition effects in task switching: Evidence from switching cue modality suggests episodic binding and response inhibition. Psychological research, 82(3), 570-579.

Kreidler et al. (2013). GLIMMPSE: Online power computation for linear models with and without a baseline covariate. Journal of Statistical Software, 54(10): i10.

Kwak, H. W., \& Egeth, H. (1992). Consequences of allocating attention to locations and to other attributes. Perception \& Psychophysics, 51(5), 455-464

Law, M. B., Pratt, J., \& Abrams, R. A. (1995). Color-based inhibition of return. Perception \& Psychophysics, 57(3), 402-408.

Lupianez, J. (2010). Inhibition of return. In A. C. Nobre \& J. T. Coull (Eds)., Attention and time (pp. 17-34). Oxford: Oxford University Press.

Lupiáñez, J., Martín-Arévalo, E., \& Chica, A. B. (2013). Is Inhibition of Return due to attentional disengagement or to a detection cost? The Detection Cost Theory of IOR. Psicologica: International Journal of Methodology and Experimental Psychology, 34(2), 221-252.

Lupiáñez, J., \& Milliken, B. (1999). Inhibition of return and the attentional set for integrating versus differentiating information. The Journal of General Psychology, 126(4), 392-418.

Lupianez, J., Ruz, M., Funes, M. J., \& Milliken, B. (2007). The manifestation of attentional capture: facilitation or IOR depending on task demands. Psychological Research, 71(1), 77-91.
MacInnes, W. J., Krüger, H. M., \& Hunt, A. R. (2015). Just passing through? Inhibition of return in saccadic sequences. Quarterly Journal of Experimental Psychology, 68(2), 402-416.

MacPherson, A. C., Klein, R. M., \& Moore, C. (2003). Inhibition of return in children and adolescents. Journal of Experimental Child Psychology, 85(4), 337-351.

Martin-Arevalo, E., Chica, A. B., \& Lupianez, J. (2016). No single electrophysiological marker for facilitation and inhibition of return: a review. Behavioural brain research, 300, 1-10.

Martín-Arévalo, E., Lupiáñez, J., Narganes-Pineda, C., Marino, G., Colás, I., \& Chica, A. B. (2019). The causal role of the left parietal lobe in facilitation and inhibition of return. Cortex, 117, 311-322.

Maylor, E. A. (1985). Facilitatory and inhibitory componets of orienting in visual space. In M.I. Posner \& O. S. Marin (Eds.), Attention and Performance XI (pp. 189-204). Hillsdale, NJ., Lawrence Erlbaum Associates Inc.

Maylor, E. A., \& Hockey, R. (1985). Inhibitory component of externally controlled covert orienting in visual space. Journal of Experimental Psychology: Human Perception and Performance, 11, 777-787.

Memelink, J., \& Hommel, B. (2013). Intentional weighting: a basic principle in cognitive control. Psychological Research, 77(3), 249-259.

Milliken, B., Joordens, S., Merikle, P. M., \& Seiffert, A. E. (1998). Selective attention: A reevaluation of the implications of negative priming. Psychological review, 105(2), 203-229.

Moeller, B., \& Frings, C. (2014). Attention meets binding: Only attended distractors are used for the retrieval of event files. Attention, Perception, \& Psychophysics, 76(4), 959-978.

Müller, H. J., \& Krummenacher, J. (2006). Locus of dimension weighting: Preattentive or postselective?. Visual Cognition, 14(48), 490-513.

Notebaert, W., \& Soetens, E. (2003). The influence of irrelevant stimulus changes on stimulus and response repetition effects. Acta Psychologica, 112(2), 143-156.

Pascucci, D., Mancuso, G., Santandrea, E., Della Libera, C., Plomp, G., \& Chelazzi, L. (2019). Laws of concatenated perception: Vision goes for novelty, decisions for perseverance. PLoS biology, 17(3), e3000144.

Pashler, H., \& Baylis, G. C. (1991). Procedural learning: II. Intertrial repetition effects in speeded-choice tasks. Journal of Experimental Psychology: Learning, Memory, and Cognition, 17(1), 33-48.

Posner, M. I. (1980). Orienting of attention. Quarterly journal of experimental psychology, 32(1), 3-25.

Posner, M. I., \& Cohen, Y. (1984). Components of visual orienting. In H. Bouma \& D.G. Bouwhuis (Eds.), Attention and Performance X: Control of language processes (pp. 531-556). Hove, UK: Lawrence Erlbaum Associated LTD.

Posner, M. I., Rafal, R. D., Choate, L., Vaughan, J. (1985). Inhibition of return: neural basis and function. Cognitive Neuropsychologia, 2, 211-228.

Pratt, J., \& Abrams, R. A. (1999). Inhibition of return in discrimination tasks. Journal of Experimental Psychology: Human Perception and Performance, 25(1), 229-242.

Pratt, J., Adam, J., \& O'Donnell, C. (2005). Response selection influences inhibition of return. European Journal of Cognitive Psychology, 17(3), 319-328.

Pratt, J., \& Fischer, M. H. (2002). Examining the role of the fixation cue in inhibition of return. Canadian Journal of Experimental Psychology/Revue canadienne de psychologie expérimentale, 56(4), 294-301.

Prime, D. J., \& Jolicoeur, P. (2009a). Response-selection conflict contributes to inhibition of return. Journal of Cognitive Neuroscience, 21(5), 991-999.

Prime, D. J., \& Jolicœur, P. (2009b). On the relationship between occipital cortex activity and inhibition of return. Psychophysiology, 46(6), 1278-1287. 
Prime, D. J., Visser, T. A., \& Ward, L. M. (2006). Reorienting attention and inhibition of return. Perception \& Psychophysics, 68(8), 13101323.

Proctor, R. W., \& Vu, K-P. L. (2006). Stimulus-response compatibility principles: Data, theory, and application. Boca Raton, FL: Taylor \& Francis CRC Press

Rajsic, J., Bi, Y., \& Wilson, D. E. (2014). Long-term facilitation of return: A response-retrieval effect. Psychonomic bulletin \& review, 21(2), 418-424.

Sanders, A. (1998). Elements of Human Performance Reaction Processes and Attention in Human Skill. New Jersey: Lawrence Erlbaum.

Schöpper, L. M., Hilchey, M. D., Lappe, M., \& Frings, C. (2019). Dataset for: Detection versus Discrimination: The Limits of Binding Accounts in Action Control.

Schumacher, E. H., \& Hazeltine, E. (2016). Hierarchical task representation: Task files and response selection. Current Directions in Psychological Science, 25(6), 449-454.

Singh, T., Moeller, B., Koch, I., \& Frings, C. (2018). May I have your attention please: Binding of attended but response-irrelevant features. Attention, Perception, \& Psychophysics, 80(5), 1143-1156.

Soetens, E., Boer, L. C., \& Hueting, J. E. (1985). Expectancy or automatic facilitation? Separating sequential effects in two-choice reaction time. Journal of Experimental Psychology: Human Perception and Performance, 11(5), 598-616.

Soetens, E. (1998). Localizing sequential effects in serial choice reaction time with the information reduction procedure. Journal of Experimental Psychology: Human Perception and Performance, 24(2), 547-568.

Tanaka, Y., \& Shimojo, S. (1996). Location vs feature: Reaction time reveals dissociation between two visual functions. Vision research, 36(14), 2125-2140.

Tanaka, Y., \& Shimojo, S. (2000). Repetition priming reveals sustained facilitation and transient inhibition in reaction time. Journal of Experimental Psychology: Human Perception and Performance, 26(4), 1421-1435.

Taylor, T. L., \& Donnelly, M. P. (2002). Inhibition of return for target discriminations: The effect of repeating discriminated and irrelevant stimulus dimensions. Perception \& Psychophysics, 64(2), 292-317.
Taylor, T. L., \& Ivanoff, J. (2005). Inhibition of return and repetition priming effects in localization and discrimination tasks. Canadian Journal of Experimental Psychology/Revue canadienne de psychologie expérimentale, 59(2), 75-89.

Taylor, T. L., \& Klein, R. M. (2000). Visual and motor effects in inhibition of return. Journal of Experimental Psychology: Human Perception and Performance, 26(5), 1639-1656.

Terry, K. M., Valdes, L. A., \& Neill, W. T. (1994). Does "inhibition of return" occur in discrimination tasks?. Perception \& psychophysics, 55(3), 279-286.

Theeuwes, J. (2019). Goal-Driven, Stimulus-Driven and History-Driven selection. Current opinion in psychology, 97-101.

Töllner, T., Gramann, K., Müller, H. J., Kiss, M., \& Eimer, M. (2008). Electrophysiological markers of visual dimension changes and response changes. Journal of Experimental Psychology: Human Perception and Performance, 34(3), 531-542.

Valyear, K. F., Fitzpatrick, A. M., \& Dundon, N. M. (2019). Now and then: Hand choice is influenced by recent action history. Psychonomic bulletin \& review, 26(1), 305-314.

Welsh, T. N., \& Pratt, J. (2006). Inhibition of return in cue-target and target-target tasks. Experimental brain research, 174(1), 167-175.

Wilson, D. E., Castel, A. D., \& Pratt, J. (2006). Long-term inhibition of return for spatial locations: Evidence for a memory retrieval account. The Quarterly Journal of Experimental Psychology, 59(12), 21352147.

Yashar, A., White, A. L., Fang, W., \& Carrasco, M. (2017). Feature singletons attract spatial attention independently of feature priming. Journal of vision, 17(9), 7-7.

Zehetleitner, M., Rangelov, D., Muller, H. (2012). Partial repetition costs persist in nonsearch compound tasks: Evidence for multipleweighting-systems hypothesis. Attention, Perception \& Psychophysics, 74, 879-890.

Publisher's note Springer Nature remains neutral with regard to jurisdictional claims in published maps and institutional affiliations. 